\title{
Weighted Clément operator and application to the finite element discretization of the axisymmetric Stokes problem
}

\author{
Zakaria Belhachmi • Christine Bernardi • \\ Simone Deparis
}

Received: 29 October 2003 / Revised: 8 October 2004 /

Published online: 20 October 2006

(C) Springer-Verlag 2006

\begin{abstract}
We consider the Stokes problem in an axisymmetric three-dimensional domain with data which are axisymmetric and have angular component equal to zero. We observe that the solution is also axisymmetric and the velocity has also zero angular component, hence the solution satisfies a system of equations in the meridian domain. The weak three-dimensional problem reduces to a two-dimensional one with weighted integrals. The latter is discretized by Taylor-Hood type finite elements. A weighted Clément operator is defined and approximation results are proved. This operator is then used to derive the discrete inf-sup condition and optimal a priori error estimates.
\end{abstract}

Mathematics Subject Classification $35 \mathrm{~N} 30 \cdot 35 \mathrm{Q} 30 \cdot 46 \mathrm{E} 35 \cdot 6 \mathrm{~N} 10 \cdot 76 \mathrm{D} 07$

\section{Introduction}

Numerical simulation of three-dimensional flows with finite elements may feature a very high computational complexity. Reducing the dimension of the problem is sometimes of paramount interest. A simple approach consists of

\footnotetext{
Z. Belhachmi

LMAM, UMR 7122, Univ. de Metz, ISGMP, Ile du Saulcy,

57045 Metz Cedex 01, France

e-mail: belhach@poncelet.univ-metz.fr

C. Bernardi

Laboratoire Jacques-Louis Lions, C.N.R.S. et Univ. Pierre et Marie Curie,

B.C. 187, 4 pl. Jussieu, 75252 Paris Cedex 05, France

e-mail: bernardi@ann.jussieu.fr

S. Deparis $(\square)$

CMCS-IACS, EPFL, 1015 Lausanne, Suisse

e-mail: simone.deparis@epfl.ch
} 
using Stokes equations in two dimensions and solve them with finite elements. This majorly reduces the size of the problem, but several three-dimensional features are not present in the model. If the problem is set in a domain which is symmetric by rotation around an axis, it is proved in [4] that, when using a Fourier expansion with respect to the angular variable, the three-dimensional problem is equivalent to a system of two-dimensional problems on the meridian domain, each problem being satisfied by a Fourier coefficient of the solution. So it is possible to reduce its size without losing three-dimensional features.

Here we are going to present an axisymmetric model which supposes data with angular component equal to zero. The advantage is that its discretization results in a linear system of the same size as a two-dimensional one. In this case, all the Fourier coefficients of the solution but the one of order zero vanish. So the number of unknowns in its discretization is the same as in the Cartesian twodimensional one. The only further difficulty is that the variational formulation requires weighted Sobolev spaces.

For the discretization of the Stokes problem we have chosen to work with Taylor-Hood finite elements, also called P1isoP2/P1 elements: The approximation of the pressure makes use of continuous piecewise affine functions and the approximation of the velocity relies also on continuous piecewise affine functions but on a finer mesh. We refer to $[14,16]$ for the numerical analysis of the discretization by other types of finite elements in a similar framework.

As usual, the numerical analysis of the discrete problem relies on an inf-sup condition of Babuška [1] and Brezzi [6] type. For the analogous discretization of the two-dimensional Stokes problem in the Cartesian case, Bercovier and Pironneau in [2] prove an inf-sup condition and Verfürth in [15] refines the analysis of these elements. Our aim is to extend these results to the axisymmetric case.

The proof of the inf-sup condition in [15] needs a very accurate approximation property of the discrete spaces, involving both the usual Lagrange interpolation operator and the Clément projection operator (see [8]). One of the main parts of this paper is devoted to the extension of the properties of these operators to the weighted Sobolev spaces. A first work in this subject is due to Mercier and Raugel (see [13]). However the results therein are not sufficient for our needs.

Once these results are established we prove an optimal inf-sup condition between discrete spaces and optimal a priori error estimates.

An extension of these results to Navier-Stokes equations may be found in [9] and an other extension to the case of non-axisymmetric solutions issued from non-axisymmetric data is under consideration.

The outline of the paper is as follows:

- In Sect. 2 we introduce the basic assumptions and the Stokes problem in the axisymmetric framework. Then we recall the definition of the weighted Sobolev spaces and some of their properties. This allows to write the variational formulation of the Stokes problem in the meridian domain. At the end of the section, the finite element formulation is presented. 
- In Sect. 3 we prove weighted inverse inequalities for the finite element functions.

- Sect. 4 is devoted to the definition of weighted Clément operators and the proof of some of their fundamental properties.

- In Sect. 5 we prove the discrete inf-sup condition related to the Taylor-Hood elements and a priori error estimates.

\section{Axisymmetric Stokes equations}

In this section we introduce the model, the notation and we recall some results from [4].

\subsection{Model description}

We are interested in modeling a flow through a domain $\breve{\Omega}$ symmetric with respect to the $z$ axis (see Fig. 1). We use cylindrical coordinates $(r, \theta, z)$ and we note $\Omega$ the half section $(r, 0, z)$. On the boundary $\breve{\Gamma}$ of the physical domain $\breve{\Omega}$ we impose a Dirichlet boundary condition. $\Gamma$ notes the half section of $\breve{\Gamma}$ and $\Gamma_{0}$ the intersection of $\breve{\Omega}$ with the axis, such that $\partial \Omega$ is the union of $\Gamma$ and $\Gamma_{0}$. All vector fields on $\breve{\Omega}$ are expressed in cylindrical coordinates.

The fluid is modeled by Stokes equations in the domain $\breve{\Omega}$ and we suppose that the boundary condition and the external forces are axisymmetric and that their angular component is zero.

Scalar functions $\breve{p}$ or vector fields $\breve{\boldsymbol{u}}$ on $\breve{\Omega}$ are axisymmetric (with respect to the $z$-axis) if for any rotation $\mathcal{R}_{\eta}$ around the $z$-axis and arbitrary angle $\eta$ in $[-\pi, \pi)$, it holds
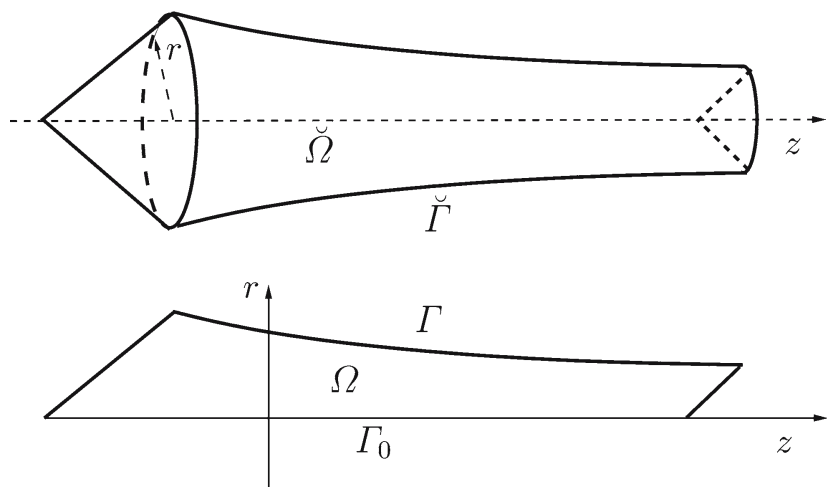

Fig. 1 The axisymmetric domain $\breve{\Omega}$ and its half section $\Omega$ 


$$
\begin{aligned}
\breve{p} \circ \mathcal{R}_{\eta} & =\breve{p}, \\
\mathcal{R}_{-\eta}\left(\breve{\boldsymbol{u}} \circ \mathcal{R}_{\eta}\right) & =\breve{\boldsymbol{u}} .
\end{aligned}
$$

In particular each cylindrical component of $\breve{\boldsymbol{u}}$ is also axisymmetric.

An axisymmetric function $\breve{p}$ on $\breve{\Omega}$ depends only on the radial and axial coordinates, therefore we associate a function $p$ on $\Omega$ such that $p(r, z)=$ $\breve{p}(r, 0, z)$. An axisymmetric vector field $\breve{\boldsymbol{u}}$ depends on $(r, z)$. For any vector field $\breve{\boldsymbol{u}}$, we denote by $\breve{u}_{r}, \breve{u}_{\theta}, \breve{u}_{z}$ its radial, angular and axial components. If it has zero angular component $\left(\breve{u}_{\theta}=0\right)$, we associate a vector field $\boldsymbol{u}=\left(u_{r}, u_{z}\right)$ on $\Omega$ such that $u_{r}=\breve{u}_{r}$ and $u_{z}=\breve{u}_{z}$.

\subsection{The Stokes problem and its weak form}

Suppose that the axisymmetric domain $\breve{\Omega}$ is bounded, has a Lipschitzcontinuous boundary, that $\Gamma_{0}$ is a finite union of segments of positive length and that the data are axisymmetric with zero angular component.

The stationary homogeneous three-dimensional Stokes problem reads

$$
\begin{cases}-v \Delta \breve{\boldsymbol{u}}+\nabla \breve{p}=\breve{\boldsymbol{f}} & \text { in } \breve{\Omega}, \\ \operatorname{div} \breve{\boldsymbol{u}}=0 & \text { in } \breve{\Omega}, \\ \breve{\boldsymbol{u}}=\mathbf{0} & \text { on } \partial \breve{\Omega},\end{cases}
$$

where $\breve{\boldsymbol{f}}$ is in $H^{-1}(\breve{\Omega})^{3}$. For simplicity we have chosen to work with zero boundary data, however the further analysis extends without difficulty to axisymmetric boundary data $\breve{g}$ with zero angular component and zero flux through $\partial \breve{\Omega}$. Differential equation (1) writes in weak form as:

Find $(\breve{\boldsymbol{u}}, \breve{p})$ in $H_{0}^{1}(\breve{\Omega})^{3} \times L_{0}^{2}(\breve{\Omega})$ such that for all $(\breve{\boldsymbol{v}}, \breve{q})$ in $H_{0}^{1}(\breve{\Omega})^{3} \times L_{0}^{2}(\breve{\Omega})$

$$
\left\{\begin{array}{l}
\breve{a}(\breve{\boldsymbol{u}}, \breve{\boldsymbol{v}})+\breve{b}(\breve{\boldsymbol{v}}, \breve{p})=\int_{\breve{\Omega}} \breve{\boldsymbol{f}} \cdot \breve{\boldsymbol{v}} \mathrm{d} \breve{\boldsymbol{x}}, \\
\breve{b}(\breve{\boldsymbol{u}}, \breve{q})=0
\end{array}\right.
$$

where the bilinear forms $\breve{a}$ and $\breve{b}$ are defined as

$$
\begin{aligned}
& \breve{a}(\breve{\boldsymbol{u}}, \breve{\boldsymbol{v}})=v \int_{\breve{\Omega}}(\nabla \breve{\boldsymbol{u}}: \nabla \breve{\boldsymbol{v}}) \mathrm{d} \breve{\boldsymbol{x}}, \\
& \breve{b}(\breve{\boldsymbol{u}}, \breve{q})=-\int_{\breve{\Omega}}(\operatorname{div} \breve{\boldsymbol{u}}) \breve{q} \mathrm{~d} \breve{\boldsymbol{x}},
\end{aligned}
$$

$H_{0}^{1}(\breve{\Omega})$ stands for the space of functions in $H^{1}(\breve{\Omega})$ with zero trace and $L_{0}^{2}(\breve{\Omega})$ for the space of functions in $L^{2}(\breve{\Omega})$ with integral equal to zero.

Bernardi et al. have shown in [4, Sect. IX.1] that this problem has a unique axisymmetric solution and that it can be split in two separate problems on $\Omega$, one for the angular component $\breve{u}_{\theta}$ and the other for $\left(\breve{u}_{r}, \breve{u}_{z}, p\right)$. If the data have 
no rotation as supposed, i.e., the angular component $\breve{f}_{\theta}$ is equal to zero, then $\breve{u}_{\theta}$ is also zero.

\subsection{Weighted Sobolev spaces}

In this section we introduce some weighted Sobolev spaces (see [12] and [4, Sect. II.1]) that we use in the weak formulation of the problem.

For any real number $\alpha$ and $1 \leqslant p<\infty$, the space $L_{\alpha}^{p}(\Omega)$ is defined as the set of measurable functions $w$ such that

$$
\|w\|_{L_{\alpha}^{p}(\Omega)}=\left(\int_{\Omega}|w|^{p} r^{\alpha} \mathrm{d} \boldsymbol{x}\right)^{\frac{1}{p}}<\infty
$$

where $r=r(\boldsymbol{x})$ is the radial coordinate of $\boldsymbol{x}$, i.e., the distance of a point $\boldsymbol{x}$ in $\Omega$ from the symmetry axis and $\mathrm{d} \boldsymbol{x}=\mathrm{d} r \mathrm{~d} z$. For $p=\infty, L_{\alpha}^{\infty}(\Omega)$ is simply equal to $L^{\infty}(\Omega)$. The subspace $L_{1,0}^{2}(\Omega)$ of $L_{1}^{2}(\Omega)$ denotes the functions $q$ with weighted integral equal to zero:

$$
\int_{\Omega} q r \mathrm{~d} \boldsymbol{x}=0 .
$$

Let $\ell$ be a positive integer. We define the weighted Sobolev space $W_{1}^{\ell, p}(\Omega)$ as the space of functions in $L_{1}^{p}(\Omega)$ such that their partial derivatives of order less or equal to $\ell$ belong to $L_{1}^{p}(\Omega)$. The space $W_{1}^{\ell, p}(\Omega)$ is a Banach space endowed with the following semi-norm $|\cdot|_{W_{1}^{\ell, p}(\Omega)}$ and norm $\|\cdot\|_{W_{1}^{\ell, p}(\Omega)}$ :

$$
\begin{aligned}
|w|_{W_{1}^{\ell, p}(\Omega)} & =\left(\sum_{k=0}^{\ell}\left\|\partial_{r}^{k} \partial_{z}^{\ell-k} w\right\|_{L_{1}^{p}(\Omega)}^{p}\right)^{\frac{1}{p}}, \\
\|w\|_{W_{1}^{\ell, p}(\Omega)} & =\left(\sum_{k=0}^{\ell}|w|_{W_{1}^{k, p}(\Omega)}^{p}\right)^{\frac{1}{p}} .
\end{aligned}
$$

For any positive real number $s, W_{1}^{s, p}(\Omega)$ is defined as the interpolation space of index $[s]+1-s$ between the spaces $W_{1}^{[s]+1, p}(\Omega)$ and $W_{1}^{[s], p}(\Omega)$, where $[s]$ is the integral part of $s$.

When $p=2$, we note as in the standard case $W_{1}^{\ell, 2}(\Omega)$ by $H_{1}^{\ell}(\Omega)$. We also need another weighted space $V_{1}^{1}(\Omega)$ defined as

$$
V_{1}^{1}(\Omega)=\left\{w \in H_{1}^{1}(\Omega) ; w \in L_{-1}^{2}(\Omega)\right\}
$$

and endowed with the norm 


$$
\|w\|_{V_{1}^{1}(\Omega)}=\left(|w|_{H_{1}^{1}(\Omega)}^{2}+\|w\|_{L_{-1}^{2}(\Omega)}^{2}\right)^{\frac{1}{2}}
$$

It can be proved that all functions in $V_{1}^{1}(\Omega)$ have a null trace on $\Gamma_{0}$ (see [13]). The traces on $\Gamma$ are defined in a nearly standard way, see [3, Sect. I, Theorem a.5]. Let $H_{1}^{s}(\Gamma), s \geqslant 0$, be the scale of Sobolev spaces built from

$$
L_{1}^{2}(\Gamma)=\left\{g: \Gamma \rightarrow \mathbb{R} \text { measurable } ; \int_{\Gamma} g^{2} r(t) \mathrm{d} t<\infty\right\},
$$

where $r(t)$ is the value of the radial coordinate at the point with tangential coordinate $t$. The trace operator $\left.v \mapsto v\right|_{\Gamma}$ is continuous from $H_{1}^{1}(\Omega)$ onto $H_{1}^{\frac{1}{2}}(\Gamma)$ and also from $V_{1}^{1}(\Omega)$ into the same space.

\subsection{Dimension reduction}

In this section we are going to underline the correspondence of the standard three-dimensional and weighted two-dimensional Sobolev spaces. See [4, Sect. II.4] for the proofs of the following statements.

The subspace of axisymmetric functions in $H^{1}(\breve{\Omega})$ is isomorphic to $H_{1}^{1}(\Omega)$. In the original three-dimensional problem, to take into account the boundary condition, the subspace $H_{0}^{1}(\breve{\Omega})$ of zero trace functions is introduced. As explained in [4, Sect. II.4], the counterpart for the axial component of the velocity is the weighted subspace

$$
H_{1 \diamond}^{1}(\Omega)=\left\{w \in H_{1}^{1}(\Omega) ; w=0 \text { on } \Gamma\right\}
$$

and the one for the radial component is

$$
V_{1 \diamond}^{1}(\Omega)=\left\{w \in V_{1}^{1}(\Omega) ; w=0 \text { on } \Gamma\right\}
$$

We describe the axisymmetric domain $\breve{\Omega}$ with cylindrical coordinates $(r, \theta, z)$. It is possible to define two isomorphisms, which maps axisymmetric functions and vector-fields on $\breve{\Omega}$ to functions and vector-fields on $\Omega$. These isomorphisms are called reduction operators and are defined in the scalar case as

$$
\begin{aligned}
\left\{\breve{w} \in H_{0}^{1}(\breve{\Omega}) \text { axisymmetric }\right\} & \longrightarrow \quad H_{1 \diamond}^{1}(\Omega), \\
\breve{w} & \longmapsto w: w(r, z)=\breve{w}(r, \theta, z)
\end{aligned}
$$


and in the vector case as

$$
\begin{aligned}
\left\{\breve{\boldsymbol{w}} \in H_{0}^{1}(\breve{\Omega})^{3} \text { axisym. and } \breve{w}_{\theta}=0\right\} & \longrightarrow V_{1 \diamond}^{1}(\Omega) \times H_{1 \diamond}^{1}(\Omega), \\
\breve{\boldsymbol{w}} & \longmapsto \boldsymbol{w}: w_{r}=\breve{w}_{r}, w_{z}=\breve{w}_{z} .
\end{aligned}
$$

Proposition 1 The space of axisymmetric vector fields in $H^{1}(\breve{\Omega})^{3}$ with zero angular component is isomorphic to $V_{1}^{1}(\Omega) \times H_{1}^{1}(\Omega)$. The space of axisymmetric vector fields in $H_{0}^{1}(\breve{\Omega})^{3}$ with zero angular component is isomorphic to $V_{1 \diamond}^{1}(\Omega) \times H_{1 \diamond}^{1}(\Omega)$.

We refer to [4, Sect. II.4] and to [9] for its proof.

\subsection{The weak axisymmetric form}

The Stokes problem (2) on $\Omega$ for $\left(\breve{u}_{r}, \breve{u}_{z}, p\right)$ is equivalent to the following weak formulation of the Stokes axisymmetric problem.

Find $(\boldsymbol{u}, p)$ in $V_{1 \diamond}^{1}(\Omega) \times H_{1 \diamond}^{1}(\Omega) \times L_{1,0}^{2}(\Omega)$ such that, for all $(\boldsymbol{v}, q)$ in $V_{1 \diamond}^{1}(\Omega) \times$ $H_{1 \diamond}^{1}(\Omega) \times L_{1,0}^{2}(\Omega)$,

$$
\left\{\begin{array}{l}
a(\boldsymbol{u}, \boldsymbol{v})+b(\boldsymbol{v}, p)=\int_{\Omega} \boldsymbol{f} \cdot \boldsymbol{v} r \mathrm{~d} \boldsymbol{x} \\
b(\boldsymbol{u}, q)=0
\end{array}\right.
$$

where the forms $a$ and $b$ are defined by

$$
\begin{aligned}
& a(\boldsymbol{u}, \boldsymbol{v})=v \int_{\Omega}\left(\nabla_{a} \boldsymbol{u}: \nabla_{a} \boldsymbol{v}\right) r \mathrm{~d} \boldsymbol{x}+v \int_{\Omega} u_{r} v_{r} \frac{1}{r} \mathrm{~d} \boldsymbol{x}, \\
& b(\boldsymbol{u}, q)=-\int_{\Omega}\left(\operatorname{div}_{a} \boldsymbol{u}\right) q r \mathrm{~d} \boldsymbol{x}-\int_{\Omega} u_{r} q \mathrm{~d} \boldsymbol{x},
\end{aligned}
$$

where $\nabla_{a} \boldsymbol{u}$ is equal to $\left(\begin{array}{ll}\partial_{r} u_{r} & \partial_{r} u_{z} \\ \partial_{z} u_{r} & \partial_{z} u_{z}\end{array}\right)$ and $\operatorname{div}_{a} \boldsymbol{u}=\partial_{r} u_{r}+\partial_{z} u_{z}$ (the index $a$ in this notation means "axisymmetric"). Indeed it can be checked that $a(\boldsymbol{u}, \boldsymbol{v})=$ $\frac{1}{2 \pi} \breve{a}(\breve{\boldsymbol{u}}, \breve{v})$ and $b(\boldsymbol{u}, q)=\frac{1}{2 \pi} \breve{b}(\breve{\boldsymbol{u}}, \breve{q})$.

In [4, Sect. IX.1] it is proved that this problem has a unique solution. In particular it is easily derived from its analogue on $\breve{\Omega}$ by using the reduction operator that the following inf-sup condition holds: There exists a constant $\beta$ such that for all $q$ in $L_{1,0}^{2}(\Omega)$,

$$
\sup _{\boldsymbol{v} \in V_{1 \diamond}^{1}(\Omega) \times H_{1 \diamond}^{1}(\Omega)} \frac{b(\boldsymbol{v}, q)}{\|\boldsymbol{v}\|_{V_{1}^{1}(\Omega) \times H_{1}^{1}(\Omega)}} \geqslant \beta\|q\|_{L_{1}^{2}(\Omega)} .
$$


Fig. 2 The mesh on the half section $\Omega$ and its axisymmetric representation in $\breve{\Omega}$

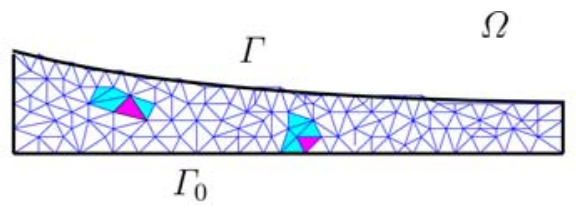

$\breve{\Omega}$

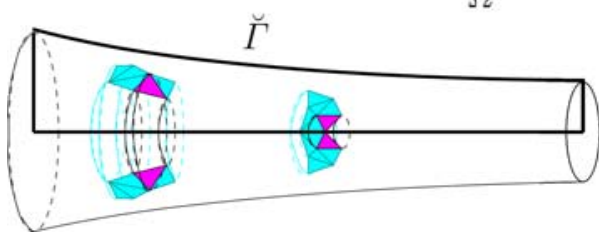

\subsection{Finite element formulation}

In this section we introduce the finite element that we employ to numerically solve the Stokes problem.

The half section $\Omega$ represents the computational domain (see Fig. 2). From now on we suppose that the domain $\Omega$ is polygonal and we introduce a regular family of triangulations $\left(\mathcal{T}_{h}\right)_{h}$ of $\Omega$ with the following properties:

(i) The domain $\bar{\Omega}$ is the union of the elements of $\mathcal{T}_{h}$.

(ii) $T_{k} \cap T_{j}$ is a side, a node or empty for all triangles $T_{k}, T_{j}, k \neq j$, in $\mathcal{T}_{h}$.

(iii) There exists a constant $\sigma$ independent of $h$, such that for all $T$ in $\mathcal{T}_{h}$, its diameter $h_{T}$ is smaller than $h$ and $T$ contains a circle of radius $\sigma h_{T}$.

We also suppose that each triangle $T$ in $\mathcal{T}_{h}$ has at least one vertex inside $\Omega$ (not on $\left.\Gamma \cup \Gamma_{0}\right)$. In all that follows, $c, c^{\prime}, \ldots$, denote generic constants that may depend on $\sigma$ and vary from one line to the next one but are always independent of $h$.

Each triangulation $\mathcal{T}_{h}$ is used for P1 elements for the pressure. Moreover $\mathcal{T}_{h / 2}$ denotes the triangulation obtained from $\mathcal{T}_{h}$ by dividing each triangle into four equal triangles by joining the midpoints of the edges. Indeed $\mathcal{T}_{h / 2}$ is used for P1 elements for the velocity.

Let $P_{k}(T)$ denote the set of restrictions to $T$ of polynomials of degree less than or equal to $k$; then the finite element spaces for the velocity and the pressure are

$$
\begin{aligned}
V_{h / 2} & =\left\{\boldsymbol{v}_{h} \in \mathcal{C}^{0}(\bar{\Omega})^{2}:\left.\boldsymbol{v}_{h}\right|_{\Gamma}=0,\left.v_{h, r}\right|_{\Gamma_{0}}=0 ;\left.\forall T \in \mathcal{T}_{h / 2} \boldsymbol{v}_{h}\right|_{T} \in P_{1}(T)^{2}\right\}, \\
Q_{h} & =\left\{q_{h} \in \mathcal{C}^{0}(\bar{\Omega}): \int_{\Omega} q_{h} r \mathrm{~d} \boldsymbol{x}=0 ;\left.\forall T \in \mathcal{T}_{h} q_{h}\right|_{T} \in P_{1}(T)\right\} .
\end{aligned}
$$

It is easily checked that $V_{h / 2} \subset V_{1 \diamond}^{1}(\Omega) \times H_{1 \diamond}^{1}(\Omega)$ and $Q_{h} \subset L_{1,0}^{2}(\Omega)$. Then the discrete problem associated with formulation (3) reads: 
Find $\left(\boldsymbol{u}_{h}, p_{h}\right)$ in $V_{h / 2} \times Q_{h}$ such that for all $\left(\boldsymbol{v}_{h}, q_{h}\right)$ in $V_{h / 2} \times Q_{h}$

$$
\left\{\begin{array}{l}
a\left(\boldsymbol{u}_{h}, \boldsymbol{v}_{h}\right)+b\left(\boldsymbol{v}_{h}, p_{h}\right)=\int_{\Omega} \boldsymbol{f} \cdot \boldsymbol{v}_{h} r \mathrm{~d} \boldsymbol{x}, \\
b\left(\boldsymbol{u}_{h}, q\right)=0 .
\end{array}\right.
$$

\section{Weighted inverse inequalities}

\subsection{Preliminary results}

In this section we are going to prove the inverse inequality for vector fields in $V_{h / 2}$. We need the following classification of the triangles. For any $T$ in $\mathcal{T}_{h}$, let $F_{T}$ denote an affine mapping from a reference triangle $\hat{T}$ onto $T$. Then the vertices of $\hat{T}, \hat{\boldsymbol{a}}_{i}, i=1,2,3$, are mapped by $F_{T}$ onto the vertices of $T, \boldsymbol{a}_{i}, i=1,2,3$. Let $\hat{\lambda}_{i}$ be the barycentric coordinate associated with $\hat{\boldsymbol{a}}_{i}$. We also define a scalar number $r_{T}$ which is the minimum of the radial coordinate of the vertices of $T$ not belonging to the axis.

Lemma 1 For any triangle $T$ of $\mathcal{T}_{h}$, there exists a constant $c$, such that $c h_{T} \leqslant r_{T}$.

Proof Since the family of triangulations is regular, the distance of a vertex $(r, z)$ to the opposite side of $T$ is larger or equal to $2 \sigma h_{T}$. Since $r_{T}$ is the distance of a vertex from the axis and no triangle crosses the axis, $r_{T} \geqslant 2 \sigma h_{T}$. The only exception is when the vertex is on a triangle which crosses the axis and has a side on $\Gamma$. In this case $r_{T} \geqslant C h_{T}$, where $C$ depends on the angles between $\Gamma$ and $\Gamma_{0}$. Since there is a finite number of intersections between $\Gamma$ and $\Gamma_{0}$, the constant $C$ is bounded from below by a positive constant.

From now on and for brevity, we say that two nonnegative functions $f_{1}$ and $f_{2}$ on a domain $T$ are equivalent if there exist two constants $c$ and $c^{\prime}$, called equivalence constants, such that the inequalities $c f_{1}(\boldsymbol{x}) \leq f_{2}(\boldsymbol{x}) \leq c^{\prime} f_{1}(\boldsymbol{x})$ hold for almost every $\boldsymbol{x}$ in $T$. It can be noted that, on all triangles $T$ of $\mathcal{T}_{h}$, the constant functions $\max _{\boldsymbol{x} \in T} r(\boldsymbol{x})$ and $r_{T}$ are equivalent. For more precise results, we observe that the triangles $T$ of $\mathcal{T}_{h / 2}$ can be parted into three types:

- Type 1. If $T \cap \Gamma_{0}$ is empty, the ratio $\frac{\max _{\boldsymbol{x} \in T} r(\boldsymbol{x})}{\min _{x \in T} r(\boldsymbol{x})}$ is smaller than a constant only depending on the regularity parameter $\sigma$ of the family of triangulations. Then $r$ is equivalent to $r_{T}$.

- Type 2. If $T \cap \Gamma_{0}$ is an edge, for instance with endpoints $\boldsymbol{a}_{2}$ and $\boldsymbol{a}_{3}$, the function $r$ is equal to $\alpha_{1}^{T} \lambda_{1}$, with the constant $\alpha_{1}^{T}$ equal to $r_{T}$, so that the ratio $\alpha_{1}^{T} / h_{T}$ is bounded from above and from below by positive constants only depending on $\sigma$.

- Type 3. If $T \cap \Gamma_{0}$ is a vertex, for instance $\boldsymbol{a}_{1}$, the function $r$ is equal to $\alpha_{2}^{T} \lambda_{2}+\alpha_{3}^{T} \lambda_{3}$, with the constants $\alpha_{i}^{T}$ equal to $r\left(\boldsymbol{a}_{i}\right)$, so that the ratio $\alpha_{i}^{T} / h_{T}$ is bounded from above and from below by positive constants only depending on $\sigma$. So, the function $r$ is equivalent to $h_{T}\left(\lambda_{2}+\lambda_{3}\right)$, with equivalence constants only depending on $\sigma$. 
Here we introduce some technical lemmas which will be used to establish the approximation properties of the Lagrange interpolation operator and the Clément operator.

We fix an integer $k \geqslant 1$ and, with each $T$ in $\mathcal{T}_{h}$, we associate its lattice of order $k$. Let $\Sigma_{h}=\left\{\boldsymbol{a}_{i}, 1 \leqslant i \leqslant N_{h}\right\}$ be the union of these lattices on all $T$ in $\mathcal{T}_{h}$.

Lemma 2 Let $\varphi_{i}$ denote the Lagrange function in $P_{k}(T)$ associated to the node $\boldsymbol{a}_{i}=\left(r_{i}, z_{i}\right)$ of $\Sigma_{h}$. Then there exists a constant $c$ independent of $h_{T}$, such that for all $T$ in $\mathcal{T}_{h}$ containing $\boldsymbol{a}_{i}$ the following inequalities hold

$$
\left\|\varphi_{i}\right\|_{L_{1}^{p}(T)} \leqslant c\left(\max _{T} r^{\frac{1}{p}}\right) h_{T}^{\frac{2}{p}}, \quad\left\|\varphi_{i}\right\|_{W_{1}^{1, p}(T)} \leqslant c\left(\max _{T} r^{\frac{1}{p}}\right) h_{T}^{\frac{2}{p}-1} .
$$

Proof Since the proof is similar for both inequalities we only give it for the first one. Indeed, it is readily checked by going to the reference element that

$$
\left\|\varphi_{i}\right\|_{L_{1}^{p}(T)} \leqslant\left(\max _{T} r^{\frac{1}{p}}\right) h_{T}^{\frac{2}{p}}\left\|\hat{\varphi}_{i}\right\|_{L^{p}(\hat{T})}
$$

Note that if $T$ intersect $\Gamma_{0}$ then $r_{T}$ and $\max _{x \in T} r$ are of the same order as $h_{T}$.

\subsection{Inverse inequalities}

Firstly inequalities are proved for the norm of $L_{-1}^{p}(T)$, then the semi-norm of $W_{1}^{\ell, p}(T)$ and finally the proof is carried out in the norms of $V_{1}^{1}(\Omega) \times H_{1}^{1}(\Omega)$.

For a triangle $T$ in $\mathcal{T}_{h}$, note its area by $|T|$. Let $f$ be a polynomial defined in $T$, then $\hat{f}$ stands for $f \circ F_{T}$. In particular $\rho_{T}=r \circ F_{T}$ is the affine function representing the radial coordinate.

Lemma 3 Let $1 \leqslant p<\infty$ and $k$ be an integer. There exists a constant $c$, such that for every triangle $T$ in $\mathcal{T}_{h}$ and any polynomial $f$ in $P_{k}(T)$, vanishing on the axis if $T$ is of type 2 or 3 ,

$$
\|f\|_{L_{-1}^{p}(T)} \leqslant c r_{T}^{-2 / p}\|f\|_{L_{1}^{p}(T)} .
$$

Proof If $T$ is of type 1, then for any point in $T, r_{T} \leqslant r$ and

$$
\int_{T}|f|^{p} \frac{1}{r} \mathrm{~d} \boldsymbol{x} \leqslant \frac{1}{r_{T}^{2}} \int_{T}|f|^{p} r \mathrm{~d} \boldsymbol{x} .
$$

Let $T$ be of type 2. On the reference triangle, the following weighted norms on the polynomials $\hat{f}$ of degree $k$ are equivalent, in particular

$$
\left\|\hat{f} \hat{\lambda}_{1}^{-1 / p}\right\|_{L^{p}(\hat{T})} \leqslant c|| \hat{f} \hat{\lambda}_{1}^{1 / p} \|_{L^{p}(\hat{T})}
$$


Hence inequality (8) is proved by

$$
\begin{aligned}
\|f\|_{L_{-1}^{p}(T)}^{p} & =\frac{|T|}{2}\left\|\hat{f}\left(\hat{\lambda}_{1} r_{T}\right)^{-1 / p}\right\|_{L^{p}(\hat{T})}^{p} \\
& \leqslant c \frac{|T|}{2} \frac{1}{r_{T}^{2}}\left\|\hat{f}\left(\hat{\lambda}_{1} r_{T}\right)^{1 / p}\right\|_{L^{p}(\hat{T})}^{p}=c \frac{1}{r_{T}^{2}}\|f\|_{L_{1}^{p}(T)}^{p} .
\end{aligned}
$$

If $T$ is of type 3, from Eq. (9) with $\hat{\lambda}_{1}$ replaced by $\hat{\lambda}_{2}+\hat{\lambda}_{3}$,

$$
\begin{aligned}
\|f\|_{L_{-1}^{p}(T)}^{p} & \leqslant c \frac{|T|}{2}\left\|\hat{f}\left(\left(\hat{\lambda}_{2}+\hat{\lambda}_{3}\right) r_{T}\right)^{-1 / p}\right\|_{L^{p}(\hat{T})}^{p} \\
& \leqslant c^{\prime} \frac{|T|}{2} \frac{1}{r_{T}^{2}}\left\|\hat{f}\left(\left(\hat{\lambda}_{2}+\hat{\lambda}_{3}\right) r_{T}\right)^{1 / p}\right\|_{L^{p}(\hat{T})}^{p} \leqslant c^{\prime} \frac{1}{r_{T}^{2}}\|f\|_{L_{1}^{p}(T)}^{p} .
\end{aligned}
$$

Lemma 4 There exists a constant $c$, such that for every triangle $T$ in $\mathcal{T}_{h}$ and any polynomial $f$ in $P_{k}(T)$,

$$
\left\|\nabla_{a} f\right\|_{L_{1}^{p}(T)} \leqslant c h_{T}^{-1}\|f\|_{L_{1}^{p}(T)} .
$$

Proof If $T$ is of type 1, the standard inverse inequality gives

$$
\begin{aligned}
\left\|\nabla_{a} f\right\|_{L_{1}^{p}(T)} & \leqslant \max _{\boldsymbol{x} \in T} r(\boldsymbol{x})^{\frac{1}{p}}\left\|\nabla_{a} f\right\|_{L^{p}(T)} \leqslant c\left(\max _{\boldsymbol{x} \in T} r(\boldsymbol{x})\right)^{\frac{1}{p}} h_{T}^{-1}\|f\|_{L^{p}(T)} \\
& \leqslant c\left(\frac{\max _{\boldsymbol{x} \in T} r(\boldsymbol{x})}{\min _{\boldsymbol{x} \in T} r(\boldsymbol{x})}\right)^{\frac{1}{p}} h_{T}^{-1}\|f\|_{L_{1}^{p}(T)},
\end{aligned}
$$

and the boundedness of the quantity $\max _{\boldsymbol{x} \in T} r(\boldsymbol{x}) / \min _{\boldsymbol{x} \in T} r(\boldsymbol{x})$ leads to the desired inequality. If $T$ is of type 2 , we have by using the transformation $F_{T}$

$$
\left\|\nabla_{a} f\right\|_{L_{1}^{p}(T)} \leq c h_{T}^{\frac{2}{p}-1} r_{T}^{\frac{1}{p}}\left\|(\nabla \hat{f}) \hat{\lambda}_{1}^{\frac{1}{p}}\right\|_{L^{p}(\hat{T})},
$$

and, by using the equivalence of norms on a finite-dimensional space, we obtain

$$
\left\|\nabla_{a} f\right\|_{L_{1}^{p}(T)} \leq c h_{T}^{\frac{2}{p}-1} r_{T}^{\frac{1}{p}}\left\|\hat{f} \hat{\lambda}_{1}^{\frac{1}{p}}\right\|_{L^{p}(\hat{T})} .
$$

Thus going back to $T$ yields

$$
\left\|\nabla_{a} f\right\|_{L_{1}^{p}(T)} \leq c h_{T}^{-1}\|f\|_{L_{1}^{p}(T)},
$$

which is the desired result. When $T$ is of type 3 , the inequality follows from the same argument as previously, with $\hat{\lambda}_{1}$ replaced by $\hat{\lambda}_{2}+\hat{\lambda}_{3}$. 
Now we are ready to prove a weighted inverse inequality:

Proposition 2 There exists a constant c such that for all $\boldsymbol{v}_{h}$ in $V_{h / 2}$,

$$
\left\|\boldsymbol{v}_{h}\right\|_{V_{1}^{1}(\Omega) \times H_{1}^{1}(\Omega)}^{2} \leqslant c \sum_{T \in \mathcal{T}_{h / 2}} h_{T}^{-2}\left\|\boldsymbol{v}_{h}\right\|_{L_{1}^{2}(T)^{2}}^{2}
$$

Proof Let $\boldsymbol{v}_{h}=\left(v_{r}, v_{z}\right)$ be in $V_{h / 2}$. From Lemmas 1 and 3, for any $T$ in $\mathcal{T}_{h / 2}$,

$$
\left\|v_{r}\right\|_{L_{-1}^{2}(T)} \leqslant c h_{T}^{-1}\left\|v_{r}\right\|_{L_{1}^{2}(T)}
$$

and from Lemma 4

$$
\left\|\nabla_{a} v_{r}\right\|_{L_{1}^{2}(T)} \leqslant c h_{T}^{-1}\left\|v_{r}\right\|_{L_{1}^{2}(T)}
$$

and this last inequality holds also for $v_{z}$.

\section{Weighted approximation properties}

We now prove some properties of the Lagrange interpolation operator and also of some Clément type operators. There are several possible constructions of the Clément operator, we follow here the approach presented in [5, Sect. IX.3] in the Cartesian case. We begin by some technical lemmas which are useful in what follows.

\subsection{Preliminary results}

The next lemma states a polynomial approximation property which is a weighted extension of a result due to [10] in a more general but unweighted case.

For $\boldsymbol{a}_{i}$ in $\Sigma_{h}, \widetilde{\Delta}_{i}$ denotes the union of two triangles containing $\boldsymbol{a}_{i}$ and sharing a common edge, and $h_{i}$ stands for its diameter.

Lemma 5 For all $p, 1 \leqslant p \leqslant+\infty$, there exists a constant $c$, independent of $h_{i}$, such that for all function in $W_{1}^{1, p}\left(\widetilde{\Delta}_{i}\right)$,

$$
\inf _{q \in P_{0}\left(\widetilde{\Delta}_{i}\right)}\left(\|v-q\|_{L_{1}^{p}\left(\widetilde{\Delta}_{i}\right)}+h_{i}|v-q|_{W_{1}^{1, p}\left(\widetilde{\Delta}_{i}\right)}\right) \leqslant c h_{i}|v|_{W_{1}^{1, p}\left(\widetilde{\Delta}_{i}\right)} .
$$

Proof Let $T$ and $T^{\prime}$ denote the two triangles which define $\widetilde{\Delta}_{i}$, and $e$ their common edge. Let $h_{e}$ denote the diameter of $e$ and $\boldsymbol{m}_{e}$ its midpoint. There exists a constant $\lambda$ depending only on the regularity parameter $\sigma$, such that $\widetilde{\Delta}_{i}$ is star shaped with respect to the ball $B$ centered on $\boldsymbol{m}_{e}$ and with radius $\frac{\lambda h_{e}}{2}$. The 
function : $\boldsymbol{x} \mapsto \hat{\boldsymbol{x}}=2 \frac{\boldsymbol{x}-\boldsymbol{m}_{e}}{\lambda h_{e}}$, from $\widetilde{\Delta}_{i}$ into a region $\hat{\Delta}$ maps the ball $B$ into the unit ball $\hat{B}$. Let $\hat{\varphi}$ be in $\mathcal{D}(\hat{B})$, with $\int_{\hat{B}} \hat{\varphi} d \hat{\boldsymbol{x}}=1$, then the function $\varphi$ defined by

$$
\varphi(\boldsymbol{x})=\left(\frac{\lambda h_{e}}{2}\right)^{-2} \hat{\varphi}\left(2 \frac{\boldsymbol{x}-\boldsymbol{m}_{e}}{\lambda h_{e}}\right)
$$

belongs to $\mathcal{D}(B)$ and

$$
\int_{B} \varphi \mathrm{d} \boldsymbol{x}=1
$$

Define $q$ as

$$
q=\int_{B} \varphi(\boldsymbol{y}) v(\boldsymbol{y}) \mathrm{d} \boldsymbol{y}
$$

To evaluate the norm of $v-q$ in $L_{1}^{p}\left(\widetilde{\Delta}_{i}\right)$, we start with the following Taylor formula: for each $\boldsymbol{x} \in \widetilde{\Delta}_{i}$, and $\boldsymbol{y} \in B$,

$$
v(\boldsymbol{x})=v(\boldsymbol{y})+\int_{0}^{1}(\boldsymbol{x}-\boldsymbol{y}) \cdot \nabla_{a} v(\boldsymbol{x}+s(\boldsymbol{y}-\boldsymbol{x})) \mathrm{d} s .
$$

Multiplying by $\varphi(\boldsymbol{y})$ and integrating over $B$, we obtain

$$
v(\boldsymbol{x})-q=\int_{B} \int_{0}^{1} \varphi(\boldsymbol{y})(\boldsymbol{x}-\boldsymbol{y}) \cdot \nabla_{a} v(\boldsymbol{x}+s(\boldsymbol{y}-\boldsymbol{x})) \mathrm{d} s \mathrm{~d} \boldsymbol{y} .
$$

Setting $\boldsymbol{z}=\boldsymbol{x}+s(\boldsymbol{y}-\boldsymbol{x})$ yields

$$
|v(\boldsymbol{x})-q| \leqslant \int_{\bar{\Delta}_{i}}\left|\int_{0}^{1} \varphi\left(\boldsymbol{x}+s^{-1}(\boldsymbol{z}-\boldsymbol{x})\right) s^{-1}(\boldsymbol{x}-\boldsymbol{z}) \cdot \nabla_{a} v(\boldsymbol{z}) s^{-2} \mathrm{~d} s\right| \mathrm{d} \boldsymbol{z},
$$

whence, for any $\boldsymbol{x}$ in $\widetilde{\Delta}_{i}$,

$$
|v(\boldsymbol{x})-q| \leqslant \int_{\bar{\Delta}_{i}}\left|k(\boldsymbol{x}, \boldsymbol{z})(\boldsymbol{x}-\boldsymbol{z}) \cdot\left(\nabla_{a} v\right)(\boldsymbol{z})\right| \mathrm{d} \boldsymbol{z},
$$


where

$$
k(\boldsymbol{x}, \boldsymbol{z})=\int_{0}^{1} \varphi\left(\boldsymbol{x}+s^{-1}(\boldsymbol{z}-\boldsymbol{x})\right) s^{-3} \mathrm{~d} s .
$$

Since $\varphi\left(\boldsymbol{x}+s^{-1}(\boldsymbol{z}-\boldsymbol{x})\right)$ vanishes when $\left|\boldsymbol{x}+s^{-1}(\boldsymbol{z}-\boldsymbol{x})-\boldsymbol{m}_{e}\right| \geqslant \frac{\lambda h_{e}}{2}$, and particularly for $s \leqslant\left(\mu h_{e}\right)^{-1}|\boldsymbol{z}-\boldsymbol{x}|$, for a constant $\mu$ depending only on $\sigma$,

$$
\begin{aligned}
|k(\boldsymbol{x}, \boldsymbol{z})| & \leqslant\|\varphi\|_{L^{\infty}(B)} \int_{\left(\mu h_{e}\right)^{-1}|\boldsymbol{z}-\boldsymbol{x}|}^{1} s^{-3} \mathrm{~d} s \\
& \leqslant c\|\varphi\|_{L^{\infty}(B)}\left(\mu h_{e}\right)^{2}|| \boldsymbol{x}-\left.\boldsymbol{z}\right|^{-2}-\left(\mu h_{e}\right)^{-2} \mid .
\end{aligned}
$$

Using $\|\varphi\|_{L^{\infty}(B)}=\left(\frac{\lambda h_{e}}{2}\right)^{-2}\|\hat{\varphi}\|_{L^{\infty}(\hat{B})}$, we deduce

$$
|k(\boldsymbol{x}, \boldsymbol{z})| \leqslant c\left(|\boldsymbol{x}-\boldsymbol{z}|^{-2}+\left(\mu h_{e}\right)^{-2}\right) .
$$

Let $\tilde{k}$ be the function

$$
\tilde{k}(\boldsymbol{z})=\left(\left|\boldsymbol{z}-\boldsymbol{m}_{e}\right|^{-2}+\left(\mu h_{e}\right)^{-2}\right)\left|\boldsymbol{z}-\boldsymbol{m}_{e}\right|
$$

and deduce from (14) and (15)

$$
|(v-q)(\boldsymbol{x})| \leqslant c\left(\tilde{k} *\left|\nabla_{a} v\right|\right)(\boldsymbol{x})
$$

We now check that, for a constant $c$ only depending on the regularity parameter $\sigma$,

$$
r(z) \geqslant \operatorname{cr}(\boldsymbol{x}) \text {. }
$$

Indeed,

- either the intersection of $\tilde{\Delta}_{i}$ with $\Gamma_{0}$ is empty. Then, we have

$$
r(\boldsymbol{z}) \geqslant \frac{\min _{\boldsymbol{t} \in \tilde{\Delta}_{i}} r(\boldsymbol{t})}{\max _{\boldsymbol{t} \in \tilde{\Delta}_{i}} r(\boldsymbol{t})} r(\boldsymbol{x}),
$$

and the ratio $\min _{\boldsymbol{t} \in \tilde{\Delta}_{i}} r(\boldsymbol{t}) / \max _{\boldsymbol{t} \in \tilde{\Delta}_{i}} r(\boldsymbol{t})$ is bounded from below by a constant only depending on $\sigma$,

- or it is not empty. We note that

$$
r(\boldsymbol{z})=(1-s) r(\boldsymbol{x})+\operatorname{sr}(\boldsymbol{y}) \geqslant \min \{r(\boldsymbol{x}), r(\boldsymbol{y})\} .
$$


Since $r(\boldsymbol{y}) \geqslant \mu h_{e}$, either $r(\boldsymbol{x}) \leqslant \mu h_{e}$, so that $r(\boldsymbol{z}) \geqslant r(\boldsymbol{x})$, or $r(\boldsymbol{x})>\mu h_{e}$, so that $r(\boldsymbol{z}) \geqslant \mu h_{e}$ and, since $r(\boldsymbol{x}) \leqslant c h_{e}$ for a constant $c$ only depending on $\sigma$, $r(\boldsymbol{z}) \geqslant \frac{\mu}{c} r(\boldsymbol{x})$.

Thus

$$
|(v-q)(\boldsymbol{x})| r(\boldsymbol{x})^{\frac{1}{p}} \leqslant c \int_{\widetilde{\Delta}_{i}} \tilde{k}(\boldsymbol{x}-\boldsymbol{z})\left|\nabla_{a} v(\boldsymbol{z})\right| r(\boldsymbol{z})^{\frac{1}{p}} \mathrm{~d} \boldsymbol{z} .
$$

Applying Young's inequality yields

$$
\|v-q\|_{L_{1}^{p}\left(\widetilde{\Delta}_{i}\right)} \leqslant\|\tilde{k}\|_{L^{1}\left(\widetilde{\Delta}_{i}\right)}\left\|\nabla_{a} v\right\|_{L_{1}^{p}\left(\widetilde{\Delta}_{i}\right)^{2}}
$$

Noting that

$$
\begin{aligned}
\|\tilde{k}\|_{L^{1}\left(\widetilde{\Delta}_{i}\right)} & =\int_{\widetilde{\Delta}_{i}}\left(\left|\boldsymbol{z}-\boldsymbol{m}_{e}\right|^{-1}+\left|\boldsymbol{z}-\boldsymbol{m}_{e}\right|\left(\mu h_{e}\right)^{-2}\right) \mathrm{d} \boldsymbol{z} \\
& \leqslant c \int_{0}^{c^{\prime} h_{e}}\left(\varrho^{-1}+\left(\mu h_{e}\right)^{-2} \varrho\right) \varrho \mathrm{d} \varrho=c^{\prime \prime} h_{e},
\end{aligned}
$$

we derive the first part of the inequality (12).

The second part of (12), i.e., the inequality with the second term in the left-hand side, is obvious.

The next lemma is an extension of the previous one and the proof is identical to the unweighted case, see [5, Sect. IX, Lemma 3.4].

Let $\Delta_{i}$ denote the union of all elements $T$ in $\mathcal{T}_{h}$ containing $\boldsymbol{a}_{i}$.

Lemma 6 For all $p, 1 \leqslant p \leqslant+\infty$, there exists a constant $c$, independent of $h_{i}$, such that, for all functions $v$ in $W_{1}^{1, p}\left(\Delta_{i}\right)$,

$$
\inf _{q \in P_{0}\left(\Delta_{i}\right)}\left(\|v-q\|_{L_{1}^{p}\left(\Delta_{i}\right)}+h_{i}|v-q|_{W_{1}^{1, p}\left(\Delta_{i}\right)}\right) \leqslant c h_{i}\|v\|_{W_{1}^{1, p}\left(\Delta_{i}\right)} .
$$

The following lemma is obtained by the same construction as Lemmas 5 and 6. Since the proof is rather long and technical, we only state the result. We refer to [10] for the analogue in the unweighted case.

Lemma 7 For all $p, 1 \leqslant p \leqslant+\infty$, there exists a constant $c$, independent of $h_{i}$, such that, for all functions $v \in W_{1}^{\ell+1, p}\left(\Delta_{i}\right)$, the following inequality holds:

$$
\inf _{q \in P_{\ell}\left(\Delta_{i}\right)}\left(\|v-q\|_{L_{1}^{p}\left(\Delta_{i}\right)}+h_{i}|v-q|_{W_{1}^{1, p}\left(\Delta_{i}\right)}\right) \leqslant c h_{i}^{\ell+1}\|v\|_{W_{1}^{\ell+1, p}\left(\Delta_{i}\right)} .
$$


Obviously the results of Lemma 5 to Lemma 7 still hold when replacing $\Delta_{i}$ by an element $T$ of $\mathcal{T}_{h}$. If we denote by $\Delta_{T}$ the union of all elements of $\mathcal{T}_{h}$ sharing at least a common vertex with $T$ then these results still hold also.

\subsection{Lagrange interpolation operator}

We define the Lagrange interpolation operator $\mathcal{I}_{h}: \mathcal{C}^{0}(\bar{\Omega}) \rightarrow X_{h}$, where $X_{h}$ denotes the space of Lagrange finite elements of order $k: \mathcal{I}_{h} \varphi$ coincides with $\varphi$ on all nodes of $\Sigma_{h}$. For any $T$ in $\mathcal{T}_{h}$, we introduce a local interpolation operator $i_{T}: \mathcal{C}^{0}(T) \rightarrow P_{k}(T)$, such that for all $\boldsymbol{a}_{j}$ in $\Sigma_{h} \cap T$,

$$
\left(i_{T} \varphi\right)\left(\boldsymbol{a}_{j}\right)=\varphi\left(\boldsymbol{a}_{j}\right) .
$$

So, it holds

$$
\mathcal{I}_{h} \varphi_{\mid T}=i_{T} \varphi
$$

Moreover this operator maps the functions that vanish on $\Gamma$ onto $X_{h} \cap H_{1 \diamond}^{1}(\Omega)$.

The approximation properties of the Lagrange interpolation operator in the framework of weighted Sobolev spaces are proved in [13] (Lemmas 6.1 and 6.2 ) in the case $p=2$ (with some restrictions). However, this is not sufficient for our purpose, and we need the more general results stated in the following proposition.

Proposition 3 For all $\ell, 1 \leqslant \ell \leqslant k+1$, and for all $p, 1 \leqslant p \leqslant+\infty$, such that

$$
\ell>\frac{3}{p} \quad \text { or } \quad p=1, \ell=3
$$

there exists a constant $C$, independent of $h$, such that, for all element $T$ in $\mathcal{T}_{h}$, the following inequalities hold for all functions $v \in W_{1}^{\ell, p}(\Omega)$ :

$$
\begin{gathered}
\left\|v-\mathcal{I}_{h} v\right\|_{L_{1}^{p}(T)} \leqslant C h_{T}^{\ell}|v|_{W_{1}^{\ell, p}(T)}, \\
\left|v-\mathcal{I}_{h} v\right|_{W_{1}^{1, p}(T)} \leqslant C h_{T}^{\ell-1}|v|_{W_{1}^{\ell, p}(T)} .
\end{gathered}
$$

Proof For any $T \in \mathcal{T}_{h}$ and for any polynomial $p$ of degree $\ell-1$,

$$
\left\|u-\mathcal{I}_{h} u\right\|_{L_{1}^{p}(T)} \leqslant\|u-p\|_{L_{1}^{p}(T)}+\left\|I_{h}(u-p)\right\|_{L_{1}^{p}(T)} .
$$

We consider the second term of this inequality. By going to the reference element, we have

$$
\left\|\mathcal{I}_{h}(u-p)\right\|_{L_{1}^{p}(T)} \leqslant c \delta h_{T}^{2 / p}\left\|\hat{\mathcal{I}}(\hat{u}-\hat{p}) \chi^{\frac{1}{p}}\right\|_{L^{p}(\hat{T})}
$$


with $\delta$ and $\chi$ defined by the type of the triangle $T$ as follows:

$$
\begin{cases}\delta=\left(\max _{x \in T} r\right)^{\frac{1}{p}}, \text { and } \chi=1 & \text { if } T \text { is of type 1, } \\ \delta=h_{T}^{\frac{1}{p}}, \text { and } \chi=\hat{\lambda}_{1} & \text { if } T \text { is of type 2, } \\ \delta=h_{T}^{\frac{1}{p}}, \text { and } \chi=\hat{\lambda}_{2}+\hat{\lambda}_{3} & \text { if } T \text { is of type 3. }\end{cases}
$$

Let $W_{\chi}^{\ell, p}(\hat{T})$ be the weighted Sobolev space with weight $\chi$ (similarly as for $\left.W_{1}^{\ell, p}(T)\right)$. The continuous embedding of $W_{\chi}^{\ell, p}(\hat{T})$ into $\mathcal{C}^{0}(\overline{\hat{T}})$, which in the first case, derives from the standard Sobolev embedding and in the other two cases from the three-dimensional one, yields

$$
\delta\|\hat{\mathcal{I}}(\hat{u}-\hat{p})\|_{L_{\chi}^{p}(\hat{T})} \leqslant \delta\|\hat{u}-\hat{p}\|_{W_{\chi}^{\ell, p}(\hat{T})}
$$

whence

$$
\left\|u-\mathcal{I}_{h} u\right\|_{L_{1}^{p}(T)} \leqslant c \delta h_{T}^{2 / p}\|\hat{u}-\hat{p}\|_{W_{\chi}^{\ell, p}(\hat{T})} .
$$

An extension of the Bramble-Hilbert lemma to the weighted case can be established by combining the compactness of the embedding $W_{\chi}^{\ell, p}(\hat{T}) \subset L_{\chi}^{p}(\hat{T})$ with the Peetre-Tartar lemma (see [11, Chapt. I, Lemma 2.1]). Using this extension, we obtain

$$
\left\|u-\mathcal{I}_{h} u\right\|_{L_{1}^{p}(T)} \leqslant c \delta h_{T}^{2 / p}|\hat{u}-\hat{p}|_{W_{\chi}^{\ell, p}(\hat{T})} \cdot
$$

Returning back to $T$ leads to (20). Inequality (21) is obtained similarly.

\subsection{The basic Clément operator}

In this section we define a regularization operator $\Pi_{h}$ which maps $L_{1}^{2}(\Omega)$ into $X_{h}$ (the space of Lagrange finite elements of order $k$ ), and we establish its approximation properties. With each $\boldsymbol{a}_{i}$ in $\Sigma_{h}$, we associate an arbitrary triangle $T_{i}$ of $\mathcal{T}_{h}$ which contains $\boldsymbol{a}_{i}$. Note that $T_{i}$ is to be chosen among a finite number of elements (bounded independently of the discretization parameter). Define $\pi_{i}$ as the $L_{1}^{2}\left(T_{i}\right)$ orthogonal projection operator onto $P_{k}\left(T_{i}\right)$ : For all $v$ in $L_{1}^{1}\left(T_{i}\right)$, $\pi_{i} v$ is in $P_{k}\left(T_{i}\right)$ and satisfies

$$
\forall q \in P_{k}\left(T_{i}\right), \quad \int_{T_{i}}\left(v-\pi_{i} v\right)(\boldsymbol{x}) q(\boldsymbol{x}) r \mathrm{~d} \boldsymbol{x}=0 .
$$


We define $\Pi_{h}$ as

$$
\Pi_{h} v=\sum_{i=1}^{N_{h}}\left(\pi_{i} v\right)\left(\boldsymbol{a}_{i}\right) \varphi_{i}(\boldsymbol{x})
$$

where $\varphi_{i}$ is the Lagrange function associated with $\boldsymbol{a}_{i}, 1 \leqslant i \leqslant N_{h}$.

The following lemma states the stability of $\pi_{i}$.

Lemma 8 For all $p, 1 \leqslant p \leqslant+\infty$, there exists a constant $c$ such that, for $1 \leqslant i \leqslant$ $N_{h}$ and for all functions $v \in L_{1}^{p}\left(T_{i}\right)$,

$$
\left\|\pi_{i} v\right\|_{L_{1}^{p}\left(T_{i}\right)} \leqslant c\|v\|_{L_{1}^{p}\left(T_{i}\right)}
$$

Proof On the reference element $\hat{T}$, we define the projection operator $\hat{\pi}$ such that $\hat{\pi} \hat{v}=\widehat{\pi_{i} v}$, namely $\hat{\pi}$ satisfies (22) with $T_{i}$ replaced by $\hat{T}$ and the measure $r \mathrm{~d} \boldsymbol{x}$ replaced by $\rho_{T_{i}}(\zeta, \eta) d \zeta d \eta$ with $\rho_{T_{i}}$ equal to $r \circ F_{T_{i}}$.

With the notation introduced in the proof of Proposition 3, the function $\rho_{T_{i}}$ is equivalent to $\delta^{p} \chi$. So we derive from Hölder's inequality that, for $p^{\prime}$ such that $\frac{1}{p}+\frac{1}{p^{\prime}}=1$,

$$
\|\hat{\pi} \hat{v}\|_{L_{\chi}^{2}(\hat{T})}^{2} \leqslant c\|\hat{v}\|_{L_{\chi}^{p}(\hat{T})}\|\hat{\pi} \hat{v}\|_{L_{\chi}^{p^{\prime}}(\hat{T})} \cdot
$$

Next, we obtain from the equivalence of weighted norms on $P_{k}(\hat{T})$ for the three weights corresponding to the different values of $\chi$ that

$$
\|\hat{\pi} \hat{v}\|_{L_{\chi}^{p}(\hat{T})} \leq c\|\hat{\pi} \hat{v}\|_{L_{\chi}^{2}(\hat{T})}, \quad\|\hat{\pi} \hat{v}\|_{L_{\chi}^{p^{\prime}(\hat{T})}} \leq c^{\prime}\|\hat{\pi} \hat{v}\|_{L_{\chi}^{2}(\hat{T})} .
$$

Combining all this gives

$$
\|\hat{\pi} \hat{v}\|_{L_{\chi}^{p}(\hat{T})} \leq c\|\hat{v}\|_{L_{\chi}^{p}(\hat{T})}
$$

Combining the previous line with the use of the transformation $F_{T_{i}}$ leads to

$$
\left\|\pi_{i} v\right\|_{L_{1}^{p}\left(T_{i}\right)} \leq c \delta\|\hat{\pi} \hat{v}\|_{L_{\chi}^{p}(\hat{T})} \leq c^{\prime} \delta\|\hat{v}\|_{L_{\chi}^{p}(\hat{T})} \leq c^{\prime \prime}\left\|\pi_{i} v\right\|_{L_{1}^{p}\left(T_{i}\right)},
$$

which is the desired result.

The following theorem states the first approximation properties of $\Pi_{h}$.

Theorem 1 For all integers $\ell, 0 \leqslant \ell \leqslant k+1$, and for all $p, 1 \leqslant p \leqslant+\infty$, there exists a constant $C$, independent of $h_{T}$, such that, for all $T \in \mathcal{T}_{h}$ and all functions $v \in W_{1}^{\ell, p}\left(\Delta_{T}\right)$, the following inequalities hold:

$$
\left\|v-\Pi_{h} v\right\|_{L_{1}^{p}(T)} \leqslant C h_{T}^{\ell}|v|_{W_{1}^{\ell, p}\left(\Delta_{T}\right)}
$$


and, when $\ell \geqslant 1$,

$$
\left|v-\Pi_{h} v\right|_{W_{1}^{1, p}(T)} \leqslant C h_{T}^{\ell-1}|v|_{W_{1}^{\ell, p}\left(\Delta_{T}\right)} .
$$

Proof The proof of (25) is divided into three cases: $\ell=0,0<\ell \leqslant \frac{3}{p}$ and $\ell>\frac{3}{p}$ (or $\ell=3, p=1$ ).

Case $\ell=0$. It holds

$$
\left\|\Pi_{h} v\right\|_{L_{1}^{p}(T)} \leqslant \sum_{i=1}^{N_{h}} \alpha_{i}\left\|\pi_{i} v\left(\boldsymbol{a}_{i}\right) \varphi_{i}\right\|_{L_{1}^{p}(T)},
$$

where $\alpha_{i}$ is equal to 1 if the intersection of the support of $\varphi_{i}$ with $T$ is not empty, and zero otherwise.

Let us fix $i$, then we can write

$$
\left\|\pi_{i} v\left(\boldsymbol{a}_{i}\right) \varphi_{i}\right\|_{L_{1}^{p}(T)} \leqslant\left\|\pi_{i} v\right\|_{L^{\infty}\left(T_{i}\right)}\left\|\varphi_{i}\right\|_{L_{1}^{p}(T)}
$$

If $T_{i}$ is of type 1 , then we obtain from Lemma 2 and a standard inverse inequality

$$
\left\|\pi_{i} v\left(\boldsymbol{a}_{i}\right) \varphi_{i}\right\|_{L_{1}^{p}(T)} \leqslant \hat{c}\left(\frac{\max _{x \in T} r}{\min _{x \in T_{i}} r}\right)^{\frac{1}{p}} h_{T_{i}}^{-\frac{2}{p}} h_{T}^{\frac{2}{p}}\left\|\pi_{i} v\right\|_{L_{1}^{p}\left(T_{i}\right)}
$$

If $T_{i}$ is of type 2, it follows from Lemma 2 and the fact that $\max _{\boldsymbol{x} \in T} r(\boldsymbol{x}) \leqslant c h_{T}$ that

$$
\left\|\varphi_{i}\right\|_{L_{1}^{p}(T)} \leqslant c h_{T}^{\frac{3}{p}}
$$

On the other hand, we have

$$
\left\|\pi_{i} v\right\|_{L^{\infty}\left(T_{i}\right)}=\left\|\widehat{\pi_{i} v}\right\|_{L^{\infty}(\hat{T})} \leqslant c\left\|\widehat{\pi_{i} v}\left(\hat{\lambda}_{1}\right)^{\frac{1}{p}}\right\|_{L^{p}(\hat{T})}
$$

whence

$$
\left\|\pi_{i} v\right\|_{L^{\infty}\left(T_{i}\right)} \leqslant c h_{T_{i}}^{-\frac{3}{p}}\left\|\pi_{i} v\right\|_{L_{1}^{p}\left(T_{i}\right)}
$$

Combining this with (29) gives

$$
\left\|\left(\pi_{i} v\right)\left(\boldsymbol{a}_{i}\right) \varphi_{i}\right\|_{L_{1}^{p}(T)} \leqslant c\left\|\pi_{i} v\right\|_{L_{1}^{p}\left(T_{i}\right)}
$$

If $T_{i}$ is of type 3 , the same arguments apply and this estimate still holds. 
Inserting (28), respectively (30), into (27), we obtain

$$
\left\|\Pi_{h} v\right\|_{L_{1}^{p}(T)} \leqslant \hat{c} \sum_{i=1}^{N_{h}} \alpha_{i}\left\|\pi_{i} v\right\|_{L_{1}^{p}\left(T_{i}\right)} .
$$

Noting that the number of non zero $\alpha_{i}$ is bounded only as a function of $k$, we deduce from Lemma 8 the inequality

$$
\left\|\Pi_{h} v\right\|_{L_{1}^{p}(T)} \leqslant c\|v\|_{L_{1}^{p}\left(\Delta_{T}\right)} \cdot
$$

Combining this with a triangle inequality yields (25) when $\ell=0$.

Case $\ell \leqslant \frac{3}{p}$. We note that for any polynomial $q \in P_{k}\left(\Delta_{T}\right)$, and for all nodes $\boldsymbol{a}_{i}$ in $T, \pi_{i} q$ is equal to $q$, therefore the restriction of $\Pi_{h} q$ to $T$ is also equal to $q$. Hence

$$
\begin{aligned}
\left\|v-\Pi_{h} v\right\|_{L_{1}^{p}(T)} & =\left\|v-q+\Pi_{h}(v-q)\right\|_{L_{1}^{p}(T)} \\
& \leqslant\|v-q\|_{L_{1}^{p}(T)}+\left\|\Pi_{h}(v-q)\right\|_{L_{1}^{p}(T)} .
\end{aligned}
$$

Using (31), we obtain

$$
\left\|v-\Pi_{h} v\right\|_{L_{1}^{p}(T)} \leqslant c\|v-q\|_{L_{1}^{p}\left(\Delta_{T}\right)}
$$

Combining with the result of Lemma 6, respectively Lemma 7 yields (25) when $\ell \leqslant \frac{3}{p}$

Case $\ell>\frac{3}{p}$ (or $\left.\ell=3, p=1\right)$. The functions of $W_{1}^{\ell, p}\left(\Delta_{T}\right)$ are continuous, therefore we can use the Lagrange interpolation operator $\mathcal{I}_{h}$. Noting that for all $\boldsymbol{a}_{i}, \pi_{i}\left(\mathcal{I}_{h} v\right)$ is equal to $\left(\mathcal{I}_{h} v\right)_{\mid T_{i}}$, we have $\Pi_{h}\left(\mathcal{I}_{h} v\right)$ which is equal to $\mathcal{I}_{h} v$. Whence

$$
\left\|v-\Pi_{h} v\right\|_{L_{1}^{p}(T)} \leqslant\left\|v-\mathcal{I}_{h} v\right\|_{L_{1}^{p}(T)}+\left\|\Pi_{h}\left(v-\mathcal{I}_{h} v\right)\right\|_{L_{1}^{p}(T)}
$$

Using once again (31) leads to

$$
\left\|v-\Pi_{h} v\right\|_{L_{1}^{p}(T)} \leqslant c\left\|v-\mathcal{I}_{h} v\right\|_{L_{1}^{p}\left(\Delta_{T}\right)}
$$

The result follows from Proposition 3.

The proof of (26) is the same as the previous one with obvious modifications (see Lemma 2).

Following the same lines we deduce the next statement.

Corollary 1 For all $\ell, 1 \leqslant \ell \leqslant k+1$, and for all $p, 1 \leqslant p \leqslant+\infty$, there exists a constant $c$, independent of $h$, such that, for all elements $T$ in $\mathcal{T}_{h}$, and all edges 
e of $T$ which are not contained in $\Gamma_{0}$, and for all functions $v \in W_{1}^{\ell, p}\left(\Delta_{T}\right)$, the following inequality holds:

$$
\left\|v-\Pi_{h} v\right\|_{L_{1}^{p}(e)} \leqslant \operatorname{ch}_{T}^{\ell-\frac{1}{p}}|v|_{W_{1}^{\ell, p}\left(\Delta_{T}\right)} .
$$

Taking the power $p$ of inequality (25) and summing it over all elements $T$, we obtain the global result

Corollary 2 For all $\ell, 1 \leqslant \ell \leqslant k+1$, and for all $p, 1 \leqslant p \leqslant+\infty$, there exists $a$ constant $c$, independent of $h$, such that, for any function $v \in W_{1}^{\ell, p}(\Omega)$,

$$
\left\|v-\Pi_{h} v\right\|_{L_{1}^{p}(\Omega)} \leqslant c h^{\ell}|v|_{W_{1}^{\ell, p}(\Omega)} \cdot
$$

\subsection{Other Clément operators}

To take into account boundary conditions, we introduce now a modified operator $\Pi_{h}^{0}$ which preserves the nullity conditions on the boundary $\Gamma$ :

$$
\Pi_{h}^{0} v=\sum_{i=1, \boldsymbol{a}_{i} \notin \Gamma}^{N_{h}}\left(\pi_{i} v\right)\left(\boldsymbol{a}_{i}\right) \varphi_{i}
$$

The operator $\Pi_{h}$ has the same approximation properties given in Theorem 1 and the proof is similar to the unweighted case, see [5, Theorem 3.11].

Corollary 3 Estimates (25), (26) and (33) still holds with $\Pi_{h}$ replaced by $\Pi_{h}^{0}$, for all functions $v$ in $W_{1}^{\ell, p}\left(\Delta_{T}\right)$ vanishing on $\Gamma \cap \Delta_{T}$.

We need also to introduce two other operators $\widetilde{\Pi}_{h}$, which maps $V_{1}^{1}(\Omega)$ into $X_{h} \cap V_{1}^{1}(\Omega)$ (the space of Lagrange finite elements of order $k$ vanishing at $\Gamma_{0}$ ), and $\widetilde{\Pi}_{h}^{0}$, which maps $V_{1 \diamond}^{1}(\Omega)$ into $X_{h} \cap V_{1 \diamond}^{1}(\Omega)$ (the space of Lagrange finite elements of order $k$ vanishing at $\Gamma_{0} \cup \Gamma$ ), defined as follows:

$$
\begin{gathered}
\widetilde{\Pi}_{h} v=\sum_{i=1, \boldsymbol{a}_{i} \notin \Gamma_{0}}^{N_{h}}\left(\pi_{i} v\right)\left(\boldsymbol{a}_{i}\right) \varphi_{i}, \\
\widetilde{\Pi}_{h}^{0} v=\sum_{i=1, \boldsymbol{a}_{i} \notin\left(\Gamma_{0} \cup \Gamma\right)}^{N_{h}}\left(\pi_{i} v\right)\left(\boldsymbol{a}_{i}\right) \varphi_{i} .
\end{gathered}
$$

Since we do not have any application for the approximation properties of these operators for all the spaces $W_{1}^{\ell, p}(\Omega)$, we restrict ourselves to the case $p=2$. We state the main result in the following theorem. 
Theorem 2 For all $\ell, 1 \leqslant \ell \leqslant k+1$, there exists a constant $c$, independent of $h$, such that, for all elements $T$ in $\mathcal{T}_{h}$, and for all functions $v \in H_{1}^{\ell}\left(\Delta_{T}\right) \cap V_{1}^{1}\left(\Delta_{T}\right)$, the following inequality holds:

$$
\left(h_{T}^{-1}\left\|v-\widetilde{\Pi}_{h} v\right\|_{L_{1}^{2}(T)}+\left\|v-\widetilde{\Pi}_{h} v\right\|_{V_{1}^{1}(T)}\right) \leqslant C h_{T}^{\ell-1}\|v\|_{H_{1}^{\ell}\left(\Delta_{T}\right) \cap V_{1}^{1}\left(\Delta_{T}\right)} .
$$

The same estimate holds with $\widetilde{\Pi}_{h}$ replaced by $\widetilde{\Pi}_{h}^{0}$ and for all $v$ in $H_{1}^{\ell}\left(\Delta_{T}\right) \cap$ $V_{1 \diamond}^{1}\left(\Delta_{T}\right)$.

Proof We consider two cases.

Case $\ell=1$. We can write

$$
\left\|\tilde{\Pi}_{h} v\right\|_{V_{1}^{1}(T)} \leqslant \sum_{i=1, a_{i} \notin \Gamma_{0}}^{N_{h}}\left\|\pi_{i} v\right\|_{L^{\infty}\left(T_{i}\right)}\left\|\varphi_{i}\right\|_{V_{1}^{1}(T)} .
$$

Using (7) and (8) for evaluating $\left\|\varphi_{i}\right\|_{V_{1}^{1}(T)}$ and the same arguments as previously for bounding $\left\|\pi_{i} v\right\|_{L^{\infty}\left(T_{i}\right)}$ according to $T_{i}$ being of type 1, 2 or 3 , we derive

$$
\left\|\tilde{\Pi}_{h} v\right\|_{V_{1}^{1}(T)} \leqslant c\|v\|_{V_{1}^{1}(T)},
$$

which yields one part of (38). On the other hand, we have

$$
\left\|v-\tilde{\Pi}_{h} v\right\|_{L_{1}^{2}(T)} \leqslant\left\|v-\Pi_{h} v\right\|_{L_{1}^{2}(T)}+\left\|\Pi_{h} v-\tilde{\Pi}_{h} v\right\|_{L_{1}^{2}(T)},
$$

and the first term in the right-hand side satisfies the desired estimate, see (25). We also note that the second term vanishes on triangles $T$ of type 1 . If $T$ is of type 2 or 3 , we derive from (7) that

$$
\left\|\Pi_{h} v-\tilde{\Pi}_{h} v\right\|_{L_{1}^{2}(T)}=\sum_{a_{i} \in \Gamma_{0} \cap T} h_{T}^{\frac{3}{2}}\left\|\pi_{i} v\right\|_{L^{\infty}\left(T_{i}\right)},
$$

where $T_{i}$ is also of type 2 or 3 . If $T_{i}$ is of type 2 for instance, we derive by the same arguments as in the proof of Lemma 8

$$
\begin{aligned}
\left\|\pi_{i} v\right\|_{L^{\infty}\left(T_{i}\right)} & =\left\|\widehat{\pi_{i} v}\right\|_{L^{\infty}(\hat{T})} \leqslant c\left\|\widehat{\pi_{i} v}\left(\hat{\lambda}_{1}\right)^{\frac{1}{2}}\right\|_{L^{\infty}(\hat{T})} \\
& \leqslant c\left\|\widehat{\pi_{i} v}\left(\hat{\lambda}_{1}\right)^{\frac{1}{2}}\right\|_{L^{2}(\hat{T})} \leqslant c\left\|\hat{v}\left(\hat{\lambda}_{1}\right)^{\frac{1}{2}}\right\|_{L^{2}(\hat{T})} .
\end{aligned}
$$

By applying the Poincaré-Friedrichs inequality to the function $\widehat{\pi_{i} v}\left(\hat{\lambda}_{1}\right)^{\frac{1}{2}}$ which vanishes on one edge of $\hat{T}$, we obtain

$$
\left\|\pi_{i} v\right\|_{L^{\infty}\left(T_{i}\right)} \leqslant c\left(\left\|\nabla \hat{v}\left(\hat{\lambda}_{1}\right)^{\frac{1}{2}}\right\|_{L^{2}(\hat{T})^{2}}+\left\|\hat{v}\left(\hat{\lambda}_{1}\right)^{-\frac{1}{2}}\right\|_{L^{2}(\hat{T})}\right) .
$$


Going back to $T_{i}$ thus gives

$$
\left\|\pi_{i} v\right\|_{L^{\infty}\left(T_{i}\right)} \leqslant c h_{T_{i}}^{1-\frac{3}{2}}\|v\|_{V_{1}^{1}\left(T_{i}\right)}
$$

The same estimate holds when $T_{i}$ is of type 3 , by using similar arguments and applying the Poincaré-Friedrichs inequality to functions in the space

$$
\hat{W}=\left\{\hat{w}\left(\hat{\lambda}_{2}+\hat{\lambda}_{3}\right)^{\frac{1}{2}} ; \hat{w} \in H^{1}(\hat{T})\right\}
$$

see [11, Chap. I, Theorem 2.1]. This concludes the proof of (38).

Case $\ell \geqslant 2$. Since $H_{1}^{\ell}(T) \subset C^{0}(\bar{T})$ and $\tilde{\Pi}_{h}\left(\mathcal{I}_{h} v\right)$ is equal to $\mathcal{I}_{h} v$ for all functions $v$ in $H_{1}^{\ell}\left(\Delta_{T}\right) \cap V_{1}^{1}\left(\Delta_{T}\right)$, we derive from inequality (38) for $\ell=1$

$$
h_{T}^{-1}\left\|v-\tilde{\Pi}_{h} v\right\|_{L_{1}^{2}(T)}+\left\|v-\tilde{\Pi}_{h} v\right\|_{V_{1}^{1}(T)} \leqslant c\left\|v-\mathcal{I}_{h} v\right\|_{V_{1}^{1}\left(\Delta_{T}\right)} .
$$

Estimate (38) follows by combining Proposition 3 with a further result proved in [13, Lemma 6.1].

To end this section, we give a useful stability property, that we state in the following theorem.

Theorem 3 There exists a constant c, independent of h, such that, for all elements $T$ in $\mathcal{T}_{h}$, and for all functions $v \in L_{-1}^{2}(T)$, the following inequality holds:

$$
\left\|\widetilde{\Pi}_{h} v\right\|_{L_{-1}^{2}(T)} \leqslant c\|v\|_{L_{-1}^{2}\left(\Delta_{T}\right)} .
$$

Proof We have

$$
\left\|\widetilde{\Pi}_{h} v\right\|_{L_{-1}^{2}(T)} \leqslant \sum_{i=1, \boldsymbol{a}_{i} \notin \Gamma_{0}}^{N_{h}}\left\|\pi_{i} v\right\|_{L^{\infty}\left(T_{i}\right)}\left\|\varphi_{i}\right\|_{L_{-1}^{2}(T)} .
$$

Combining inequalities (7), (8) and, when $T_{i}$ is of type 2 or 3 for instance,

$$
\left\|\pi_{i} v\right\|_{L^{\infty}\left(T_{i}\right)} \leqslant h_{T}^{-\frac{1}{2}}\|v\|_{L_{-1}^{2}\left(T_{i}\right)}
$$

yields (39).

As a consequence of the previous results, there exist positive constants $c$ and $c^{\prime}$ and an operator $R_{h}: V_{1 \diamond}^{1}(\Omega) \times H_{1 \diamond}^{1}(\Omega) \rightarrow V_{h / 2}$ such that for all triangles $T$ in $\mathcal{T}_{h}$ and all functions $\boldsymbol{v}$ in $V_{1 \diamond}^{1}(\Omega) \times H_{1 \diamond}^{1}(\Omega)$

$$
\begin{gathered}
\left\|\boldsymbol{v}-R_{h} \boldsymbol{v}\right\|_{L_{1}^{2}(T)^{2}} \leqslant c h_{T}\|\boldsymbol{v}\|_{V_{1}^{1}\left(\Delta_{T}\right) \times H_{1}^{1}\left(\Delta_{T}\right)}, \\
\left\|\boldsymbol{v}-R_{h} \boldsymbol{v}\right\|_{V_{1}^{1}(T) \times H_{1}^{1}(T)} \leqslant c^{\prime}\|\boldsymbol{v}\|_{V_{1}^{1}\left(\Delta_{T}\right) \times H_{1}^{1}\left(\Delta_{T}\right)} .
\end{gathered}
$$




\section{Inf-Sup condition for the Stokes problem and a priori error estimates}

5.1 Axisymmetric inf-sup condition in $\left(V_{1}^{1}(\Omega) \times H_{1}^{1}(\Omega)\right) \times H_{1}^{1}(\Omega)$

The following proposition is the weighted version of the theorem presented in [2] about the Cartesian inf-sup condition for P1isoP2/P1 elements. We also adapt it to a regular (but not necessarily uniformly regular) family of triangulations (we refer to [7, Sect. VI.6] for the idea of this extension).

Proposition 4 There exists a positive constant $c$ independent of $h$ such that

$$
\forall q_{h} \in Q_{h}, \quad \sup _{\boldsymbol{v}_{h} \in V_{h / 2}} \frac{b\left(\boldsymbol{v}_{h}, q_{h}\right)}{\left\|\boldsymbol{v}_{h}\right\|_{V_{1}^{1}(\Omega) \times H_{1}^{1}(\Omega)}} \geqslant c\left(\sum_{T \in \mathcal{T}_{h}} h_{T}^{2}\left|q_{h}\right|_{H_{1}^{1}(T)}^{2}\right)^{1 / 2} .
$$

Proof Let $q_{h}$ in $Q_{h}$ be fixed. Let $T_{k}$ and $T_{j}$ be two triangles of $\mathcal{T}_{h}$ with a common side, two common vertices denoted by $\boldsymbol{x}^{k}$ and $\boldsymbol{x}^{j}$ and let $\boldsymbol{x}^{k j}$ be their midpoint $\frac{1}{2}\left(\boldsymbol{x}^{k}+\boldsymbol{x}^{j}\right)$ (see Fig. 3). Each element $T_{k}$ of $\mathcal{T}_{h}$ being divided into four sub-triangles by joining the midpoints of its edges, let $D_{k}$ be the union of the three sub-triangles of $T_{k}$ with one vertex being $\boldsymbol{x}^{k j}$. Note $d_{k}$ the weighted measure of $D_{k}$ :

$$
d_{k}=\int_{D_{k}} r \mathrm{~d} \boldsymbol{x}
$$

Define $D_{j}$ in the same way. To simplify the notation we neglect dx in the integrals.

We define an element $\boldsymbol{v}_{h}$ of $V_{h / 2}$, being equal to zero at each vertex of any $T$ in $\mathcal{T}_{h}$ and on $\Gamma_{0} \cup \Gamma$, and equal to arbitrary real vectors $\boldsymbol{v}_{k j}$ at all midpoints $\boldsymbol{x}^{k j}$. In what follows and for brevity, we denote by $\sum_{(k j)}$ the sum over all edges with endpoints $\boldsymbol{x}_{k}$ and $\boldsymbol{x}_{j}$ which are shared by two triangles of $\mathcal{T}_{h}$. We can also write $\boldsymbol{v}_{h}$ as $\sum_{(k j)} \varphi^{k j} \boldsymbol{v}_{k j}$, where $\varphi^{k j}$ is a basis function of $V_{h / 2}$ which is one at $\boldsymbol{x}^{k j}$ and zero at the other vertices of triangles of $\mathcal{T}_{h / 2}$. The end of the proof is divided into five steps.

Step 1. The norm of $\varphi^{k j} \boldsymbol{v}_{k j}$ is bounded by

$$
\left\|\varphi^{k j} \boldsymbol{v}_{k j}\right\|_{L_{1}^{2}\left(T_{k}\right)^{2}}^{2} \leqslant \int_{D_{k}} r\left|\varphi^{k j} \boldsymbol{v}_{k j}\right|^{2} \leqslant\left|\boldsymbol{v}_{k j}\right|^{2} d_{k} .
$$

Fig. 3 The two triangles $T_{k}$ and $T_{j}$

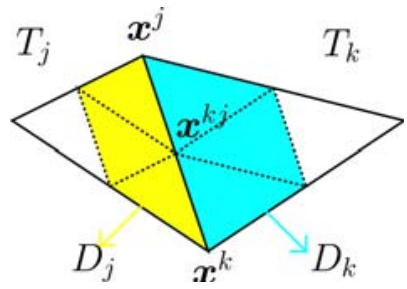


Then

$$
\left\|\boldsymbol{v}_{h}\right\|_{L_{1}^{2}(\Omega)^{2}}^{2} \leqslant \sum_{(k j)}\left\|\varphi^{k j} \boldsymbol{v}_{k j}\right\|_{L_{1}^{2}(\Omega)^{2}}^{2} \leqslant \sum_{(k j)}\left|\boldsymbol{v}_{k j}\right|^{2}\left(d_{k}+d_{j}\right) .
$$

By the inverse inequality (11) and noting $h_{k}$ the diameter $h_{T_{k}}$,

$$
\begin{aligned}
\left\|\boldsymbol{v}_{h}\right\|_{V_{1}^{1}(\Omega) \times H_{1}^{1}(\Omega)}^{2} & \leqslant c \sum_{T_{k} \in \mathcal{T}_{h}} h_{k}^{-2}\left\|\boldsymbol{v}_{h}\right\|_{L_{1}^{2}\left(T_{k}\right)^{2}}^{2} \\
& \leqslant c \sum_{T_{k} \in \mathcal{T}_{h}} \sum_{T_{j} \cap T_{k}=\text { edge }} h_{k}^{-2}\left|\boldsymbol{v}_{k j}\right|^{2} d_{k} \\
& =c \sum_{(k j)}\left(h_{k}^{-2} d_{k}+h_{j}^{-2} d_{j}\right)\left|\boldsymbol{v}_{k j}\right|^{2} \\
& \leqslant c \sigma^{-2} \sum_{(k j)} h_{k j}^{-2}\left|\boldsymbol{v}_{k j}\right|^{2}\left(d_{k}+d_{j}\right),
\end{aligned}
$$

where $h_{k j}=\max \left\{h_{k}, h_{j}\right\}$ and the last inequality holds since $T_{k}$ and $T_{j}$ have a common edge. We have shown that

$$
\left\|\boldsymbol{v}_{h}\right\|_{V_{1}^{1}(\Omega) \times H_{1}^{1}(\Omega)}^{2} \leqslant c \sum_{(k j)} h_{k j}^{-2}\left|\boldsymbol{v}_{k j}\right|^{2}\left(d_{k}+d_{j}\right) .
$$

Step 2. The vector field $\nabla_{a} q_{h}$ is constant on $T_{k}$ and it is noted by $(\nabla q)_{k}$. Let $\chi_{D}$ be the characteristic function of a set $D$, then by integration by parts

$$
b\left(\varphi^{k j} \boldsymbol{v}_{k j}, q_{h}\right)=\int_{\Omega} r \varphi^{k j} \boldsymbol{v}_{k j} \cdot \nabla_{a} q_{h}=\int_{\Omega} r \varphi^{k j} \boldsymbol{v}_{k j} \cdot\left((\nabla q)_{k} \chi_{D_{k}}+(\nabla q)_{j} \chi_{D_{j}}\right) .
$$

Now by considering the cases where $T_{k}$ is of type 1,2 or 3 and using [5, Sect. VII, Proposition 2.3], we check that there exist two positive constants $\alpha_{1}$ and $\alpha_{2}$ only depending on $\sigma$ and a scalar $\rho_{k j}$ with $\alpha_{1}<\rho_{k j}<\alpha_{2}$, such that

$$
\int_{D_{k}} \varphi^{k j} r \mathrm{~d} \boldsymbol{x}=d_{k} \rho_{k j}
$$

This implies that

$$
\int_{\Omega} r \varphi^{k j} \boldsymbol{v}_{k j} \cdot(\nabla q)_{k} \chi_{D_{k}}=\int_{D_{k}} r\left((\nabla q)_{k} \cdot \varphi^{k j} \boldsymbol{v}_{k j}\right)=\left((\nabla q)_{k} \cdot \boldsymbol{v}_{k j}\right) d_{k} \rho_{k j},
$$


hence

$$
b\left(\boldsymbol{v}_{h}, q_{h}\right)=\sum_{(k j)}\left(\rho_{k j} d_{k}(\nabla q)_{k}+\rho_{j k} d_{j}(\nabla q)_{j}\right) \cdot \boldsymbol{v}_{k j}
$$

Step 3. Equation (43) together with (45) gives

$$
\begin{aligned}
& \max _{v_{h} \in V_{h / 2}} \frac{b\left(\boldsymbol{v}_{h}, q_{h}\right)}{\left\|\boldsymbol{v}_{h}\right\|_{V_{1}^{1}(\Omega) \times H_{1}^{1}(\Omega)}} \\
& \geqslant c \max _{\left(\boldsymbol{v}_{k j}\right)(k j)}\left[\frac{\sum_{(k j)}\left(\rho_{k j} d_{k}(\nabla q)_{k}+\rho_{j k} d_{j}(\nabla q)_{j}\right) \cdot \boldsymbol{v}_{k j}}{\left(\sum_{(k j)} h_{k j}^{-2}\left|\boldsymbol{v}_{k j}\right|^{2}\left(d_{k}+d_{j}\right)\right)^{\frac{1}{2}}}\right] .
\end{aligned}
$$

We now take $\boldsymbol{v}_{k j}=h_{k j}^{2}\left(\rho_{k j} d_{k}(\nabla q)_{k}+\rho_{j k} d_{j}(\nabla q)_{j}\right) /\left(d_{k}+d_{j}\right)$, so we are in the case of equality in the Cauchy-Schwarz inequality

$$
\begin{aligned}
& \sum_{(k j)} {\left[\frac{\rho_{k j} d_{k}(\nabla q)_{k}+\rho_{j k} d_{j}(\nabla q)_{j}}{h_{k j}^{-1}\left(d_{k}+d_{j}\right)^{\frac{1}{2}}} \cdot h_{k j}^{-1} \boldsymbol{v}_{k j}\left(d_{k}+d_{j}\right)^{\frac{1}{2}}\right] } \\
&=\left[\sum_{(k j)} \frac{\left|\rho_{k j} d_{k}(\nabla q)_{k}+\rho_{j k} d_{j}(\nabla q)_{j}\right|^{2}}{h_{k j}^{-2}\left(d_{k}+d_{j}\right)}\right]^{\frac{1}{2}}\left[\sum_{(k j)} h_{k j}^{-2}\left|\boldsymbol{v}_{k j}\right|^{2}\left(d_{k}+d_{j}\right)\right]^{\frac{1}{2}} .
\end{aligned}
$$

Therefore, from (46) and (47)

$$
\max _{v_{h} \in V_{h / 2}} \frac{b\left(\boldsymbol{v}_{h}, q_{h}\right)}{\left\|\boldsymbol{v}_{h}\right\|_{V_{1}^{1}(\Omega) \times H_{1}^{1}(\Omega)}} \geqslant c\left[\sum_{(k j)} h_{k j}^{2} \frac{\left|\rho_{k j} d_{k}(\nabla q)_{k}+\rho_{j k} d_{j}(\nabla q)_{j}\right|^{2}}{d_{k}+d_{j}}\right]^{\frac{1}{2}} .
$$

Step 4. Since in $T_{k}$ the gradient $\left.\nabla_{a} q_{h}\right|_{T_{k}}=(\nabla q)_{k}$ is constant, setting $q_{k}=q_{h}\left(\boldsymbol{x}^{k}\right)$, etc., leads to

$$
(\nabla q)_{k} \cdot \frac{\boldsymbol{x}^{k}-\boldsymbol{x}^{j}}{\left|\boldsymbol{x}^{k}-\boldsymbol{x}^{j}\right|}=\frac{q_{k}-q_{j}}{\left|\boldsymbol{x}^{k}-\boldsymbol{x}^{j}\right|}
$$

and the same holds in $T_{j}$. Then

$$
\left(\rho_{k j} d_{k}(\nabla q)_{k}+\rho_{j k} d_{j}(\nabla q)_{j}\right) \cdot \frac{\boldsymbol{x}^{k}-\boldsymbol{x}^{j}}{\mid \boldsymbol{x}^{k}-\boldsymbol{x}^{j \mid}}=\frac{\left(\rho_{k j} d_{k}+\rho_{j k} d_{j}\right)\left(q_{k}-q_{j}\right)}{\left|\boldsymbol{x}^{k}-\boldsymbol{x}^{j}\right|} .
$$


Note that the $\rho_{k j}$ are larger than $\alpha_{1}$. For a vector $\boldsymbol{a}$ and a unit vector $\boldsymbol{e}$, $|\boldsymbol{a}|^{2} \geqslant|\boldsymbol{a} \cdot \boldsymbol{e}|^{2}$. Taking $e=\frac{\boldsymbol{x}^{k}-\boldsymbol{x}^{j}}{\left|\boldsymbol{x}^{k}-\boldsymbol{x}^{j}\right|}$, the square of the previous inequality gives

$$
\left(\rho_{k j} d_{k}(\nabla q)_{k}+\rho_{j k} d_{j}(\nabla q)_{j}\right)^{2} \geqslant c \frac{\left(d_{k}+d_{j}\right)^{2}\left(q_{k}-q_{j}\right)^{2}}{\left|\boldsymbol{x}^{k}-\boldsymbol{x}^{j}\right|^{2}} .
$$

Step 5. We note $\boldsymbol{x}^{l}$ the third vertex of $T_{k}$. Equality (49) and the regularity of the family of triangulations allow to write for $m=k$ and $l$,

$$
\left|(\nabla q)_{k}\right|^{2} \leqslant c\left[\frac{\left(q_{k}-q_{l}\right)^{2}}{\left|\boldsymbol{x}^{k}-\boldsymbol{x}^{l}\right|^{2}}+\frac{\left(q_{m}-q_{j}\right)^{2}}{\left|\boldsymbol{x}^{m}-\boldsymbol{x}^{j}\right|^{2}}\right] .
$$

Inequalities (48) and (50) yield

$$
\begin{aligned}
\max _{v_{h} \in V_{h / 2}} & \frac{b\left(\boldsymbol{v}_{h}, q_{h}\right)}{\left\|\boldsymbol{v}_{h}\right\|_{V_{1}^{1}(\Omega) \times H_{1}^{1}(\Omega)}} \\
& \geqslant c\left\{\sum_{T_{k} \in \mathcal{T}_{h}} \sum_{\left(\boldsymbol{x}^{k}, \boldsymbol{x}^{j}\right)}^{\text {edges of } T_{k}} h_{k j}^{2} \frac{\left(q_{k}-q_{j}\right)^{2}}{\left|\boldsymbol{x}^{k}-\boldsymbol{x}^{j}\right|^{2}}\left(d_{k}+d_{j}\right) \chi_{\left\{\boldsymbol{x}^{k j} \notin \Gamma \cup \Gamma_{0}\right\}}\right\} .
\end{aligned}
$$

Since for each triangle $T$ in $\mathcal{T}_{h}$ there is at least one vertex inside $\Omega$, in the previous equation at least two midpoints of the edges are not in $\Gamma \cup \Gamma_{0}$.

Let $t_{k}$ denote the weighted measure of the triangle $T_{k}$. Since $d_{k j} \geqslant \frac{3}{8} t_{k}$ (see [9, Appendix A1]), inequality (51) leads to

$\max _{v_{h} \in V_{h / 2}} \frac{b\left(\boldsymbol{v}_{h}, q_{h}\right)}{\left\|\boldsymbol{v}_{h}\right\|_{V_{1}^{1}(\Omega) \times H_{1}^{1}(\Omega)}} \geqslant c\left(\sum_{T_{k} \in \mathcal{T}_{h}} h_{k}^{2} t_{k}\left|(\nabla q)_{k}\right|^{2}\right)^{\frac{1}{2}}=c\left(\sum_{T \in \mathcal{T}_{h}} h_{T}^{2}\left\|\nabla_{a} q_{h}\right\|_{L_{1}^{2}(T)}^{2}\right)^{\frac{1}{2}}$.

\subsection{Axisymmetric inf-sup condition in $\left(V_{1}^{1}(\Omega) \times H_{1}^{1}(\Omega)\right) \times L_{1}^{2}(\Omega)$}

Verfürth in [15] shows the inf-sup condition for the Stokes problem with P1isoP2/P1 elements in the Cartesian case. Verfürth's argument is based on the propositions presented in the previous sections. Now we are going to state and prove the inf-sup theorem for axisymmetric $\mathrm{P} 1$ isoP2/P1 finite elements.

Theorem 4 (Axisymmetric P1isoP2/P1 inf-sup) There exists a positive constant $c$ independent of $h$ such that

$$
\forall q_{h} \in Q_{h}, \quad \sup _{\boldsymbol{v}_{h} \in V_{h / 2}} \frac{b\left(\boldsymbol{v}_{h}, q_{h}\right)}{\left\|\boldsymbol{v}_{h}\right\|_{V_{1}^{1}(\Omega) \times H_{1}^{1}(\Omega)}} \geqslant c\left\|q_{h}\right\|_{L_{1}^{2}(\Omega)} .
$$


Proof Let $q_{h}$ be in $Q_{h}$ with $\left\|q_{h}\right\|_{L_{1}^{2}(\Omega)}=1$, and denote $\eta$ as the quantity $\left(\sum_{T \in \mathcal{T}_{h}} h_{T}^{2}\left|q_{h}\right|_{H_{1}^{1}(T)}^{2}\right)^{1 / 2}$. Proposition 4 implies

$$
\sup _{\boldsymbol{v}_{h} \in V_{h / 2}} \frac{b\left(\boldsymbol{v}_{h}, q_{h}\right)}{\left\|\boldsymbol{v}_{h}\right\|_{V_{1}^{1}(\Omega) \times H_{1}^{1}(\Omega)}} \geqslant c_{1} \eta .
$$

It follows from the inf-sup condition (5) that there is a $\boldsymbol{u}$ satisfying

$$
\operatorname{div}_{a} \boldsymbol{u}+\frac{1}{r} u_{r}=-q_{h} \quad \text { and } \quad\|\boldsymbol{u}\|_{V_{1}^{1}(\Omega) \times H_{1}^{1}(\Omega)} \leqslant \beta^{-1}\left\|q_{h}\right\|_{L_{1}^{2}(\Omega)}=\beta^{-1} .
$$

This implies $\left|b\left(\boldsymbol{u}, q_{h}\right)\right|=\left\|q_{h}\right\|_{L_{1}^{2}(\Omega)}^{2}=1$. On the other hand, taking $\boldsymbol{u}_{h}=R_{h} \boldsymbol{u}$ and using (40) yields

$$
\begin{aligned}
\sum_{T \in \mathcal{T}_{h}} h_{T}^{-2}\left\|\boldsymbol{u}_{h}-\boldsymbol{u}\right\|_{L_{1}^{2}(T)}^{2} & \leqslant c^{2} \sum_{T \in \mathcal{T}_{h}}\|\boldsymbol{u}\|_{V_{1}^{1}\left(\Delta_{T}\right) \times H_{1}^{1}\left(\Delta_{T}\right)}^{2} \\
& \leqslant c^{\prime 2}\|\boldsymbol{u}\|_{V_{1}^{1}(\Omega) \times H_{1}^{1}(\Omega)}^{2} \leqslant \beta^{-2} c^{\prime 2} .
\end{aligned}
$$

By integration by parts together with the Schwarz inequality, we derive that

$$
\begin{aligned}
\left|b\left(\boldsymbol{u}_{h}-\boldsymbol{u}, q_{h}\right)\right| & =\left|\sum_{T \in \mathcal{T}_{h}} \int_{T}\left(\boldsymbol{u}_{h}-\boldsymbol{u}\right) \nabla_{a} q_{h} r \mathrm{~d} \boldsymbol{x}\right| \\
& \leqslant\left(\sum_{T \in \mathcal{T}_{h}} h_{T}^{-2}\left\|\boldsymbol{u}-\boldsymbol{u}_{h}\right\|_{L_{1}^{2}(T)}^{2}\right)^{1 / 2}\left(\sum_{T \in \mathcal{T}_{h}} h_{T}^{2}\left\|\nabla_{a} q_{h}\right\|_{L_{1}^{2}(T)}^{2}\right)^{1 / 2} \\
& \leqslant c \eta .
\end{aligned}
$$

Using (54), (41) and (55) yields

$$
\begin{aligned}
& \sup _{\boldsymbol{v}_{h} \in V_{h / 2}} \frac{b\left(\boldsymbol{v}_{h}, q_{h}\right)}{\left\|\boldsymbol{v}_{h}\right\|_{V_{1}^{1}(\Omega) \times H_{1}^{1}(\Omega)}} \\
& \geqslant \frac{b\left(\boldsymbol{u}, q_{h}\right)-\left|b\left(\boldsymbol{u}_{h}-\boldsymbol{u}, q_{h}\right)\right|}{\|\boldsymbol{u}\|_{V_{1}^{1}(\Omega) \times H_{1}^{1}(\Omega)}+\left\|\boldsymbol{u}_{h}-\boldsymbol{u}\right\|_{V_{1}^{1}(\Omega) \times H_{1}^{1}(\Omega)}} \\
& \geqslant c\left(b\left(\boldsymbol{u}, q_{h}\right)-\left|b\left(\boldsymbol{u}_{h}-\boldsymbol{u}, q_{h}\right)\right|\right) \geqslant c\left(1-c^{\prime} \eta\right)=c_{2}-c_{3} \eta .
\end{aligned}
$$


Inequalities (53) and (56) imply

$$
\begin{aligned}
\sup _{\boldsymbol{v}_{h} \in V_{h / 2}} \frac{b\left(\boldsymbol{v}_{h}, q_{h}\right)}{\left\|\boldsymbol{v}_{h}\right\|_{V_{1}^{1}(\Omega) \times H_{1}^{1}(\Omega)}} & \geqslant \max \left\{c_{1} \eta, c_{2}-c_{3} \eta\right\} \\
& \geqslant \min _{t \geqslant 0} \max \left\{c_{1} t, c_{2}-c_{3} t\right\}=\frac{c_{1} c_{2}}{c_{1}+c_{3}} .
\end{aligned}
$$

This ends the proof in the case $\left\|q_{h}\right\|_{L_{1}^{2}(\Omega)}=1$. Otherwise, if $q_{h}$ is different from zero, take $\tilde{q}_{h}=q_{h} /\left\|q_{h}\right\|_{L_{1}^{2}(\Omega)}$, which concludes the proof.

It is also possible to replace $V_{h / 2}$ by piecewise quadratic functions on $\mathcal{T}_{h}$, i.e.,

$$
\tilde{V}_{h}=\left\{\boldsymbol{v}_{h} \in \mathcal{C}^{0}(\Omega)^{2}:\left.\boldsymbol{v}_{h}\right|_{\Gamma}=0,\left.v_{h, r}\right|_{\Gamma_{0}}=0 ;\left.\forall T \in \mathcal{T}_{h} \boldsymbol{v}_{h}\right|_{T} \in P_{2}(T)^{2}\right\}
$$

The degrees of freedom of this space are exactly the same as for $V_{h / 2}$, so by the same arguments as previously, the inf-sup condition (52) still holds with $V_{h / 2}$ replaced by $\tilde{V}_{h}$.

\subsection{Existence, uniqueness and a priori error estimates}

The spaces $V_{1 \diamond}^{1}(\Omega) \times H_{1 \diamond}^{1}(\Omega)$ equipped with $\|\cdot\|_{V_{1}^{1}(\Omega) \times H_{1}^{1}(\Omega)}$ and $L_{1,0}^{2}(\Omega)$ with $\|\cdot\|_{L_{1}^{2}(\Omega)}$ are Hilbert spaces. In fact (see Proposition 1 and [4, Sect. II.2]) they are isomorphic to subspaces of $H^{1}(\breve{\Omega})^{3}$ and $L^{2}(\breve{\Omega})$ respectively. The bilinear form $a(\cdot, \cdot)$ is elliptic (property derived from $\breve{a}(\cdot, \cdot)$ ), and the bilinear form $b(\cdot, \cdot)$ satisfies by Theorem 4 the inf-sup condition. Hence the abstract results of Babuška [1], Brezzi [6] (see also [7, Sect. II.2.2] and [11, Sect. II.1]) yield the well-posedness of the discrete Stokes problem (6).

Theorem 5 Problem (6) has a unique solution $\left(\boldsymbol{u}_{h}, p_{h}\right)$ in $V_{h / 2} \times Q_{h}$. Furthermore, if $\boldsymbol{u}$ is in $H_{1}^{s+1}(\Omega)^{2}$ and $p$ in $H_{1}^{s}(\Omega), 0<s \leqslant 1$, then there exists a constant $C$ such that

$$
\left\|\boldsymbol{u}-\boldsymbol{u}_{h}\right\|_{V_{1}^{1}(\Omega) \times H_{1}^{1}(\Omega)}+\left\|p-p_{h}\right\|_{L_{1}^{2}(\Omega)} \leqslant C h^{s}\left(\|v\|_{H_{1}^{s+1}(\Omega)^{2}}+\|p\|_{H_{1}^{s}(\Omega)}\right) .
$$

Proof As pointed out in Eq. (4), the operator $a(\cdot, \cdot)$ can be expressed by the three-dimensional operator $\breve{a}(\cdot, \cdot)$, which is coercive (see for example [4, Sect. II.2]). In Sect. 5.2 the inf-sup condition (52) is proved, hence Theorem 1.1 in [11, Sect. II.1] yields

$$
\begin{aligned}
& \left\|\boldsymbol{u}-\boldsymbol{u}_{h}\right\|_{V_{1}^{1}(\Omega) \times H_{1}^{1}(\Omega)}+\left\|p-p_{h}\right\|_{L_{1}^{2}(\Omega)} \\
& \quad \leqslant C\left(\inf _{\boldsymbol{v}_{h} \in V_{h / 2}}\left\|\boldsymbol{u}-\boldsymbol{v}_{h}\right\|_{V_{1}^{1}(\Omega) \times H_{1}^{1}(\Omega)}+\inf _{q_{h} \in Q_{h}}\left\|p-q_{h}\right\|_{L_{1}^{2}(\Omega)}\right) .
\end{aligned}
$$


In the case $s=1$, Mercier and Raugel [13] in Theorem 4.4 show that the space of functions in $H_{1}^{2}(\Omega)$ vanishing on $\Gamma_{0}$ is included in $V_{1}^{1}(\Omega)$. Theorems 1 and 2 and Corollary 3 lead to

$$
\inf _{\boldsymbol{v}_{h} \in V_{h}}\left\|\boldsymbol{u}-\boldsymbol{v}_{h}\right\|_{V_{1}^{1}(\Omega) \times H_{1}^{1}(\Omega)} \leqslant C h\|v\|_{H_{1}^{2}(\Omega)^{2}}
$$

and

$$
\inf _{q_{h} \in Q_{h}}\left\|p-q_{h}\right\|_{L_{1}^{2}(\Omega)} \leqslant C h\|p\|_{H_{1}^{1}(\Omega)}
$$

which proves (57). Extending this result to the case $0<s<1$ follows from an interpolation argument (note that the inequality $\|v\|_{V_{1}^{1}(\Omega)} \leqslant c\|v\|_{H_{1}^{s+1}(\Omega)}$ is valid for all $s>0$ and for any function $v$ in $H_{1}^{s+1}(\Omega) \cap V_{1}^{1}(\Omega)$, see [3, Chap. I, Appendix]).

In particular, Bernardi et al. in [4, Sect. IX.1] show that if $\Omega$ is convex and the angles between $\Gamma$ and $\Gamma_{0}$, are not too large (for example less than $\frac{3}{4} \pi$ is enough), and if $\boldsymbol{f}$ is in $L_{1}^{2}(\Omega)^{2}$, then $\boldsymbol{u}$ is in $H_{1}^{2}(\Omega)^{2}$ and $p$ is in $H_{1}^{1}(\Omega)$ (in fact they show that $\boldsymbol{u}$ is even more regular than that). So the error behaves like $c h$ at least when these conditions on the geometry of $\Omega$ are satisfied.

Acknowledgements The third author was supported by the ETH board through a stipend for exchange between ETH Zürich and EPF Lausanne.

\section{References}

1. Babuška, I.: The finite element method with Lagrangian multipliers. Numer. Math. 20, 179-192 (1972/73)

2. Bercovier, M., Pironneau, O.: Error estimates for finite element method solution of the Stokes problem in the primitive variables. Numer. Math. 33(2), 211-224 (1979)

3. Bernardi, C., Dauge, M., Maday, Y.: Polynomials in the Sobolev World, Preprints of the Laboratories J.-L. Lions (2003)

4. Bernardi, C., Dauge, M., Maday, Y.: Spectral Methods for Axisymmetric Domains. GauthierVillars, Éditions Scientifiques et Médicales Elsevier, Paris (1999). Numerical algorithms and tests due to Mejdi Azaïez

5. Bernardi, C., Maday, Y., Rapetti, F.: Discrétisations variationnelles de problèmes aux limites elliptiques. Collection Mathématiques et Applications, Vol. 45, Springer, Berlin Heidelberg New York (2004)

6. Brezzi, F.: On the existence, uniqueness and approximation of saddle-point problems arising from Lagrangian multipliers. RAIRO Anal. Numér. 8(R-2), 129-151 (1974)

7. Brezzi, F., Fortin, M.: Mixed and Hybrid Finite Element Methods. Springer, Berlin Heidelberg New York (1991)

8. Clément, P.: Approximation by finite element functions using local regularization. RAIRO Anal. Numér. 9(R-2), 77-84 (1975)

9. Deparis, S.: Numerical analysis of axisymmetric flows and methods for fluid-structure interaction arising in blood flow simulation. PhD Thesis, École Polytechnique Fédérale de Lausanne (2004)

10. Dupont, T., Scott, R.: Polynomial approximation of functions in Sobolev spaces. Math. Comp. 34(150), 441-463 (1980) 
11. Girault, V., Raviart, P.-A.: Finite Element Methods for Navier-Stokes Equations. Theory and Algorithms. Springer, Berlin Heidelberg New York (1986)

12. Kufner, A.: Weighted Sobolev Spaces, Teubner-Texte zur Mathematik [Teubner Texts in Mathematics] Vol. 31, BSB B. G. Teubner Verlagsgesellschaft, Leipzig (1980) (with German, French and Russian summaries)

13. Mercier, B., Raugel, G.: Résolution d'un problème aux limites dans un ouvert axisymétrique par éléments finis en $r, z$ et séries de Fourier en $\theta$. RAIRO Anal. Numér. 16(4), 405-461 (1982)

14. Tabata, M.: Finite element analysis of axisymmetric flow problems. Angew. Z. Math. Mech. 76(suppl. 1), 171-174, (1996) ICIAM/GAMM 95 (Hamburg, 1995)

15. Verfürth, R.: Error estimates for a mixed finite element approximation of the Stokes equations. RAIRO Anal. Numér. 18(2), 175-182 (1984)

16. Ying, L.A.: Finite element approximation to axial symmetric Stokes flow. J. Comput. Math. 4(1), 38-49 (1986) 